\title{
Synchronization in coupled second order in time infinite-dimensional models
}

\author{
Igor Chueshov* \\ Department of Mechanics and Mathematics, \\ Karazin Kharkov National University, \\ Kharkov, 61022, Ukraine
}

August 1, 2021

\begin{abstract}
We study asymptotic synchronization at the level of global attractors in a class of coupled second order in time models which arises in dissipative wave and elastic structure dynamics. Under some conditions we prove that this synchronization arises in the infinite coupling intensity limit and show that for identical subsystems this phenomenon appears for finite intensities. Our argument involves a method based on "compensated" compactness and quasi-stability estimates. As an application we consider the nonlinear Kirchhoff, Karman and Berger plate models with different types of boundary conditions. Our results can be also applied to the nonlinear wave equations in an arbitrary dimension. We consider synchronization in sine-Gordon type models which describes distributed Josephson junctions.
\end{abstract}

Keywords: synchronization; wave dynamics; global attractor; upper semicontinuity.

MSC 2010: 37L30, 34D06, 35B41.

\section{Introduction}

Our goal in this paper is to study long-time dynamics of a class of coupled systems consisting of two second order in time evolution equations. These systems are abstract models for studies of elastic and wave dissipative dynamics in different situations. Under some set of hypotheses concerning the model we first prove the existence of finite dimensional global attractors and study their dependence on interaction operators. Then we apply these results to analyze synchronization phenomena. In this paper we understand these phenomena at the level of global attractors. This means that in synchronized regime the attractor of coupled system becomes "diagonal" in some sense.

Our main results are presented in Theorems 3.12 and 3.14 . In particular, Theorem 3.12 proves asymptotic synchronization in the limit of large coupling and Theorem 3.14 dealing with interaction of identical systems shows that synchronization is possible for finite values of coupling intensity parameter. As a preliminary step we obtain a result on uniform dissipativity with

*e-mail: chueshov@karazin.ua 
respect to coupling intensity parameters (see Theorem 3.5). We also discuss possibility of synchronization in infinite-dimensional systems by means of finite-dimensional interaction operators. As application of these results we consider a range of nonlinear elastic plate models and also wave dynamics of different types.

We note that recently the subject of synchronization of coupled (identical or not) systems has received considerable attention. There are now quite a few monographs [4, 28, 30, 34, 38, 40] in this field, which contain extensive lists of references. In the case of infinite dimensional systems the synchronization problem has been studied in [5, 7, 8, 24, 36] for coupled parabolic systems. Synchronization in Berger plates (they are a particular case of our abstract models) was considered in [31, 32, 33]. Master-slave synchronization of coupled parabolic-hyperbolic PDE systems was considered in [10, 11]. The methods involved in these publications relies either on the parabolic regularization (see [5, 8, 24]) or on a special structure of the model (see [31, 32, 33]). Our approach is different.

par As an important technical tool we involve the method developed in [19] (see also also [20, Chapter 8] and [12]) based on an observability-type estimate which allows us to establish uniform quasi-stability estimates in the case of critical nonlinearities (such as in the von Karman and Berger models). In the standard way (see, e.g., [12], 19] or [20, Chapter 8]) these quasi-stability estimates lead to appropriate uniform bounds for attractors which are important for asymptotic synchronization.

The paper is organized as follows. The next Section 2 is devoted to preliminary considerations. We describe here our abstract model, formulate main hypotheses and provide well-posedness result for rather general situation. Section 3 contains our main results on attractors and synchronization. In Section 4 we discuss possible applications. The Appendix contains some general facts from the dissipative dynamical systems theory.

\section{Preliminaries}

In this section we describe the problem and state our basic notations and hypotheses. Then we provide a well-posedness theorem which is easily derived from the known results.

\subsection{Abstract model and main hypotheses}

In a Hilbert space $H$ we deal with following coupled equations

$$
\begin{aligned}
& u_{t t}+\nu_{1} A u+D_{11} u_{t}+D_{12} v_{t}+K_{11} u+K_{12} v+B_{1}(u)=0 \\
& v_{t t}+\nu_{2} A v+D_{21} u_{t}+D_{22} v_{t}+K_{21} u+K_{22} v+B_{2}(v)=0
\end{aligned}
$$

equipped with initial data

$$
u(0)=u_{0}, \quad u_{t}(0)=u_{1}, \quad v(0)=v_{0}, \quad v_{t}(0)=v_{1},
$$

under the following set of hypotheses.

Assumption $2.1 \quad$ (i) $A$ is a self-adjoint positive operator densely defined on a domain $\mathcal{D}(A)$ in a separable Hilbert space $H, \nu_{1}, \nu_{2}>0$ are parameters. We assume that the resolvent 
of $A$ is compact in $H$. This implies that there is orthonormal basis $\left\{e_{k}\right\}$ in $H$ consisting of the eigenvectors of the operator $A$ :

$$
A e_{k}=\lambda_{k} e_{k}, \quad 0<\lambda_{1} \leq \lambda_{2} \leq \cdots, \quad \lim _{k \rightarrow \infty} \lambda_{k}=\infty .
$$

We denote by $\|\cdot\|$ and $(\cdot, \cdot)$ the norm and the scalar product in $H$. We also denote by $H^{s}$ (with $s>0$ ) the domain $\mathcal{D}\left(A^{s}\right)$ equipped with the graph norm $\|\cdot\|_{s}=\left\|A^{s} \cdot\right\| \cdot H^{-s}$ denotes the completion of $H$ with respect to the norm $\|\cdot\|_{-s}=\left\|A^{-s} \cdot\right\|$. The symbol $(\cdot, \cdot)$ denotes not only the scalar product but also the duality between $H^{s}$ and $H^{-s}$. Below we also use the notation $\bar{H}^{s}=H^{s} \times H^{s}$.

(ii) The damping operators $D_{i j}: H^{1 / 2} \mapsto H^{-1 / 2}$ are linear mappings such that the matrix operator

$$
\mathcal{D}=\left(\begin{array}{cc}
D_{11} & D_{12} \\
D_{21} & D_{21}
\end{array}\right): H^{1 / 2} \times H^{1 / 2} \mapsto H^{-1 / 2} \times H^{-1 / 2}
$$

generates a symmetric nonnegative bilinear form on $\bar{H}^{1 / 2} \equiv H^{1 / 2} \times H^{1 / 2}$.

(iii) The interaction operators $K_{i j}: H^{1 / 2} \mapsto H$ are linear and

$$
\mathcal{K}=\left(\begin{array}{ll}
K_{11} & K_{12} \\
K_{21} & K_{21}
\end{array}\right): H^{1 / 2} \times H^{1 / 2} \mapsto H \times H
$$

generates a symmetric nonnegative bilinear form on $\bar{H}^{1 / 2}$.

(iv) The nonlinear operators $B_{i}: H^{1 / 2} \rightarrow H$ are locally Lipschitz, i.e., for every $\varrho>0$ there exists a constant $L(\varrho)$ such that

$$
\left\|B_{i}(u)-B_{i}(v)\right\| \leq L(\varrho)\|u-v\|_{1 / 2}, \quad i=1,2,
$$

for all $u, v \in H^{1 / 2}$ such that $\|u\|_{1 / 2},\|v\|_{1 / 2} \leq \varrho$. In addition we assume that $B_{i}(u)$ are potential operators. This means that $B_{i}(u)=\Pi_{i}^{\prime}(u)$, where $\Pi_{i}: H^{1 / 2} \mapsto \mathbb{R}$ is a Frechét differentiable functional on $H^{1 / 2}$, i.e.,

$$
\lim _{\|v\|_{1 / 2} \rightarrow 0} \frac{1}{\|v\|_{1 / 2}}\left[\Pi_{i}(u+v)-\Pi_{i}(u)-\left(\Pi_{i}^{\prime}(u), v\right)\right]=0 .
$$

We also assume that $\Pi_{i}(u)=\Pi_{0 i}(u)+\Pi_{1 i}(u)$, where $\Pi_{0 i}(u)$ is a nonnegative locally bounded functional on $H^{1 / 2}$ and

$$
\forall \eta>0 \exists C_{\eta}: \quad\left|\Pi_{1 i}(u)\right| \leq \eta\left[\left\|A^{1 / 2} u\right\|^{2}+\Pi_{0 i}(u)\right]+C_{\eta}, \quad u \in H^{1 / 2} .
$$

The problem in (11) can be written as

$$
U_{t t}+\mathcal{A} U+\mathcal{D} U_{t}+\mathcal{K} U+\mathcal{B}(U)=0, \quad U(0)=U_{0}, \quad U_{t}(0)=U_{1},
$$

where the operators $\mathcal{D}$ and $\mathcal{K}$ are defined above and

$$
U=\left(\begin{array}{l}
u \\
v
\end{array}\right), \quad \mathcal{A}=\left(\begin{array}{cc}
\nu_{1} & 0 \\
0 & \nu_{2}
\end{array}\right) A, \quad \mathcal{B}(U)=\left(\begin{array}{c}
B_{1}(u) \\
B_{2}(v)
\end{array}\right)
$$

As it was already mentioned in Introduction our main motivation for (11) (and (2)) and also for the hypotheses in Assumption 2.1 is related with applications to coupled plate and wave systems (see Section 44). 


\subsection{Well-posedness}

Now we consider the existence and uniqueness theorem for (2). We start with adaptation of the standard definition (see, e.g, [19] and the references therein) to our model.

Definition 2.2 A function $U(t) \in C\left([0, T] ; \bar{H}^{1 / 2}\right) \cap C^{1}([0, T] ; \bar{H})$ possessing the properties $U(0)=$ $U_{0}$ and $U_{t}(0)=U_{1}$ is said to be

(S) strong solution to problem (2) on the interval $[0, T]$, iff

- $u \in W_{1}^{1}\left(a, b ; \bar{H}^{1 / 2}\right)$ and $u_{t} \in W_{1}^{1}(a, b ; \bar{H})$ for any $0<a<b<T$, where

$$
W_{1}^{1}(a, b ; H)=\left\{f \in C(a, b ; H): f^{\prime} \in L_{1}(a, b ; H)\right\},
$$

- $\mathcal{A} U(t)+D U_{t}(t) \in \bar{H}$ for almost all $t \in[0, T]$

- equation in (2) is satisfied in $\bar{H}$ for almost all $t \in[0, T]$;

(G) generalized solution to problem (2) on the interval $[0, T]$, iff there exists a sequence of strong solutions $\left\{U_{n}(t)\right\}$ with initial data $\left(U_{0 n}, U_{1 n}\right)$ instead of $\left(U_{0}, U_{1}\right)$ such that

$$
\lim _{n \rightarrow \infty} \max _{t \in[0, T]}\left\{\left\|\partial_{t} U(t)-\partial_{t} U_{n}(t)\right\|+\left\|\mathcal{A}^{1 / 2}\left(U(t)-U_{n}(t)\right)\right\|\right\}=0
$$

Application of Theorem 1.5 from [19] gives the following well-posedness result.

Theorem 2.3 Let $T>0$ be arbitrary. Under Assumption 2.1 the following statements hold.

- Strong solutions: For every $\left(U_{0} ; U_{1}\right) \in \bar{H}^{1 / 2} \times \bar{H}^{1 / 2}$, such that $\mathcal{A} U_{0}+\mathcal{D} U_{1} \in \bar{H}$ there exists unique strong solution to problem (2) on the interval $[0, T]$ such that

$$
\begin{gathered}
\left(U_{t} ; U_{t t}\right) \in L_{\infty}\left(0, T ; \bar{H}^{1 / 2} \times \bar{H}\right), \quad U_{t} \in C_{r}\left([0, T) ; \bar{H}^{1 / 2}\right), \\
U_{t t} \in C_{r}([0, T) ; \bar{H}) \quad \text { and } \quad \mathcal{A} U(t)+\mathcal{D} U_{t}(t) \in C_{r}([0, T) ; \bar{H}),
\end{gathered}
$$

where we denote by $C_{r}$ the space of right continuous functions. This solution satisfies the energy relation

$$
\mathcal{E}\left(U(t), U_{t}(t)\right)+\int_{0}^{t}\left(\mathcal{D} U_{t}(\tau), U_{t}(\tau)\right) d \tau=\mathcal{E}\left(U_{0}, U_{1}\right),
$$

where the energy $\mathcal{E}$ is defined by the relation

$$
\mathcal{E}\left(U_{0}, U_{1}\right)=\mathcal{E}_{1}\left(u_{0}, u_{1}\right)+\mathcal{E}_{2}\left(v_{0}, v_{1}\right)+\mathcal{E}_{\text {int }}\left(u_{0}, v_{0}\right),
$$

with $U_{0}=\left(u_{0} ; v_{0}\right), U_{1}=\left(u_{1} ; v_{1}\right)$,

$$
\mathcal{E}_{i}\left(u_{0}, u_{1}\right)=E_{i}\left(u_{0}, u_{1}\right)+\Pi_{i}\left(u_{0}\right) \equiv \frac{1}{2}\left(\left\|u_{1}\right\|^{2}+\nu_{i}\left\|A^{1 / 2} u_{0}\right\|^{2}\right)+\Pi_{i}\left(u_{0}\right) .
$$

and

$$
\mathcal{E}_{\text {int }}\left(u_{0}, v_{0}\right)=\frac{1}{2}\left(\mathcal{K} U_{0}, U_{0}\right)
$$


- Generalized solutions: For every $\left(U_{0} ; U_{1}\right) \in \bar{H}^{1 / 2} \times \bar{H}$ there exists unique generalized solution. This solution possesses the property $\mathcal{D}^{1 / 2} u_{t} \in L_{2}(0, T ; \bar{H})$ and satisfies the energy inequality

$$
\mathcal{E}\left(U(t), U_{t}(t)\right)+\int_{0}^{t}\left(\mathcal{D} U_{t}(\tau), U_{t}(\tau)\right) d \tau \leq \mathcal{E}\left(U_{0}, U_{1}\right)
$$

If $U^{1}$ and $U^{2}$ are generalized solutions with different initial data and $Z=U^{1}-U^{2}$, then

$$
\left\|Z_{t}(t)\right\|^{2}+\left\|\mathcal{A}^{1 / 2} Z(t)\right\|^{2}+\left\|\mathcal{K}^{1 / 2} Z(t)\right\|^{2} \leq\left(\left\|Z_{t}(0)\right\|^{2}+\left\|\mathcal{A}^{1 / 2} Z(0)\right\|^{2}+\left\|\mathcal{K}^{1 / 2} Z(t)\right\|^{2}\right) e^{a_{R} t}
$$

provided $\left\|U_{t}^{i}(0)\right\|^{2}+\left\|\mathcal{A}^{1 / 2} U^{i}(0)\right\|^{2}+\left\|\mathcal{K}^{1 / 2} U^{i}(0)\right\|^{2} \leq R^{2}$.

By Theorem 2.3 problem (2) generates a dynamical system $\left(\mathcal{H}, S_{t}\right)$ in the space

$$
\mathcal{H}=\bar{H}^{1 / 2} \times \bar{H} \equiv H^{1 / 2} \times H^{1 / 2} \times H \times H
$$

with evolution operator defined by the relation

$$
S_{t}\left(U_{0} ; U_{1}\right)=\left(U(t) ; U_{t}(t)\right)
$$

where $U(t)$ is a generalized solution to problem (2).

\section{Global Attractors}

In this section we prove the existence of a global attractor for the dynamical system $\left(\mathcal{H}, S_{t}\right)$ and study its properties. Keeping in mind further application we impose additional hypotheses concerning the damping operator $\mathcal{D}$ and the source term $\mathcal{B}$.

Assumption 3.1 Let Assumption 2.1 be in force and

(i) $\mathcal{D}$ is strictly positive, i.e. exists $c_{0}>0$ such that

$$
(\mathcal{D} W, W) \geq c_{0}\|W\|^{2}, \quad W \in \bar{H}^{1 / 2}
$$

(ii) either $B_{i}$ are subcritical, i.e., for every $\varrho>0$ there exists a constant $L(\varrho)$ such that

$$
\left\|B_{i}(u)-B_{i}(v)\right\| \leq L(\varrho)\|u-v\|_{1 / 2-\delta}, \quad i=1,2, \quad \delta>0,
$$

for all $u, v \in H^{1 / 2}$ such that $\|u\|_{1 / 2},\|v\|_{1 / 2} \leq \varrho$, or else the potential energies $\Pi_{i}$ are continuous on $H^{1 / 2-\delta}$ for some $\delta>0$ and the mapping $u \mapsto A^{-l} B_{i}(u)$ is continuous from $H^{1 / 2-\delta}$ into $H^{-l}$ for some $\delta, l>0, i=1,2$.

Proposition 3.2 Let Assumption 3.1 be in force. Then the system $\left(\mathcal{H}, S_{t}\right)$ generated by problem (2) is asymptotically smooth (see the definition in the Appendix).

Proof. We apply Theorem 3.26 and Proposition 3.36 from [19]. Since

$$
\begin{aligned}
|(\mathcal{D} V, W)| & \leq[(\mathcal{D} V, V)]^{1 / 2}[(\mathcal{D} W, W)]^{1 / 2} \leq C[(\mathcal{D} V, V)]^{1 / 2}\left\|\mathcal{A}^{1 / 2} W\right\| \\
& \leq C_{\varepsilon}(\mathcal{D} V, V)+\varepsilon\left\|\mathcal{A}^{1 / 2} W\right\|^{2},
\end{aligned}
$$

relation (3.60) in [19, p.54] obviously holds in a simplified form. 
One can see from the energy inequality in (4) that the system $\left(\mathcal{H}, S_{t}\right)$ is gradient with the full energy $\mathcal{E}\left(U_{0} ; U_{1}\right)$ as a strict Lyapunov function (see the Appendix for the corresponding definitions). Therefore by Corollary 2.29 [19] (see Theorem A.2 and Remark A.3 in the Appendix) to guarantee the existence of a global attractor we need boundedness of equilibria. This leads to the following assertion.

Theorem 3.3 Let Assumptions [3.1 be in force. Assume in addition that there exist $\nu<\nu_{i}$ and $C \geq 0$ such that

$$
\nu\left\|A^{1 / 2} u\right\|^{2}+\left(B_{i}(u), u\right)+C \geq 0, \quad u \in H^{1 / 2}, \quad i=1,2 .
$$

Then the system $\left(\mathcal{H}, S_{t}\right)$ generated by problem (2) possesses a compact global attractor.

Proof. Stationary solutions $U=(u ; v) \in \bar{H}^{1 / 2}$ solve the problem

$$
\begin{aligned}
& \nu_{1} A u+K_{12} u+K_{12} v+B_{1}(u)=0, \\
& \nu_{2} A v+K_{21} u+K_{22} v+B_{2}(v)=0 .
\end{aligned}
$$

Using the multipliers $u$ for the first equation and $v$ for the second and also positivity of the operator $\mathcal{K}$, we obtain that

$$
\nu_{1}\left\|\mathcal{A}^{1 / 2} u\right\|^{2}+\nu_{2}\left\|\mathcal{A}^{1 / 2} v\right\|^{2}+\left(B_{i}(u), u\right)+\left(B_{i}(v), v\right) \leq 0
$$

By (51) this yields $\left\|\mathcal{A}^{1 / 2} u\right\|^{2}+\left\|\mathcal{A}^{1 / 2} v\right\|^{2} \leq C$ (with $C$ independent of $\mathcal{D}$ and $\mathcal{K}$ ). Thus stationary solutions are bounded.

\subsection{Uniform dissipativity}

For synchronization phenomena it is important to have bounds for the attractor independent of interaction operators $\mathcal{D}$ and $\mathcal{K}$. In spite of the set of stationary solutions is uniformly bounded with respect to $\mathcal{D}$ and $\mathcal{K}$ Theorem 3.3 does not provide appropriate bounds for the attractor. Below we use an approach based Lyapunov type functions which allows us to prove uniform dissipativity of the system $\left(\mathcal{H}, S_{t}\right)$. To simplify argument it is convenient to introduce intensity factors $\alpha$ and $\varkappa$ for interactions in velocities and displacements. Moreover, we assume a particular structure of $\mathcal{D}$ related with interaction operator $\mathcal{K}$. So instead of (2) we consider

$$
U_{t t}+\mathcal{A} U+\left(\mathcal{D}_{0}+\alpha \mathcal{K}\right) U_{t}+\varkappa \mathcal{K} U+\mathcal{B}(U)=0, \quad U(0)=U_{0}, \quad U_{t}(0)=U_{1},
$$

where $\alpha$ and $\varkappa$ are nonnegative parameters.

In addition to Assumption 2.1] we impose the following hypotheses.

Assumption 3.4 (i) The operator $\mathcal{D}_{0}$ is bounded from $\bar{H}^{1 / 2}$ into $\bar{H}$ and there exist $c_{0}>0$ and $\bar{\alpha} \geq 0$ such that

$$
\left(\left(\mathcal{D}_{0}+\bar{\alpha} \mathcal{K}\right) W, W\right) \geq c_{0}\|W\|^{2}, \quad W \in \bar{H}^{1 / 2}
$$

(ii) there exists $\eta<1$ and $\delta, C>0$ such that

$$
\delta \bar{\Pi}_{0}(U)-(\mathcal{B}(U), U) \leq \eta\left\|\mathcal{A}^{1 / 2} U\right\|^{2}+C, \quad U \in \bar{H}^{1 / 2},
$$

where $\bar{\Pi}_{0}(U)=\Pi_{01}(u)+\Pi_{02}(v)$ with $U=(u ; v)$.

\footnotetext{
${ }^{1}$ The relation in (7) is the standard requirement in many second order in time models, see, e.g., 19.
} 
Theorem 3.5 Let Assumptions [2.1] and 3.4 be in force. Then for every $\alpha \geq \bar{\alpha}$ and $\varkappa \geq 0$ the system $\left(\mathcal{H}, S_{t}\right)$ generated by problem (6) is dissipative 2 with an absorbing ball of the size independent of $(\alpha ; \varkappa) \in \Lambda \equiv\{\alpha \geq \bar{\alpha}, \varkappa \geq 0\}$. More precisely, there exists $R$ independent of $(\alpha ; \varkappa) \in \Lambda$ such that the set

$$
\mathfrak{B}=\left\{\left(U_{0} ; U_{1}\right) \in \mathcal{H}:\left\|U_{1}\right\|^{2}+\left\|\mathcal{A}^{1 / 2} U_{0}\right\|^{2}+\varkappa\left\|\mathcal{K}^{1 / 2} U_{0}\right\|^{2} \leq R^{2}\right\}
$$

is absorbing.

We note that the estimate in (8) improves the corresponding finite-dimensional statement in [1] which requires uniform boundedness of the ratio $\alpha / \varkappa$. As it is shown in Proposition 3.8 we need the latter property for uniform quasi-stability only.

Proof. We use a slight modification of the standard method (see, e.g., [3, 9, 39]) based on Lyapunov type functions. Let $U(t)=(u(t) ; v(t))$ be a strong solution,

$$
E(t) \equiv E\left(U ; U_{t}\right)=\frac{1}{2}\left(\left\|U_{t}(t)\right\|^{2}+\left\|\mathcal{A}^{1 / 2} U(t)\right\|^{2}\right)+\bar{\Pi}(U(t))
$$

with $\bar{\Pi}(U)=\Pi_{1}(u)+\Pi_{2}(v)$, and $\Phi(t)=\eta\left(U, U_{t}\right)+\mu(\mathcal{K} U, U)$, where $\eta$ is a positive constant which will be chosen later and $2 \mu=\varkappa+\eta \alpha$. We consider the functional $V=E+\Phi$. One can see that there exist $0<\eta_{0}<1$ and $\beta_{i}>0$ independent of $(\alpha ; \varkappa)$ such that

$$
\beta_{0}\left[E_{*}(t)+\varkappa\left\|\mathcal{K}^{1 / 2} U(t)\right\|^{2}\right]-\beta_{1} \leq V \leq \beta_{2}\left[E_{*}(t)+\mu\left\|\mathcal{K}^{1 / 2} U(t)\right\|^{2}\right]+\beta_{3}
$$

for all $\eta \in\left(0, \eta_{0}\right]$, where

$$
E_{*}(t) \equiv E_{*}\left(U ; U_{t}\right)=\frac{1}{2}\left(\left\|U_{t}(t)\right\|^{2}+\left\|\mathcal{A}^{1 / 2} U(t)\right\|^{2}\right)+\bar{\Pi}_{0}(U) .
$$

Now on strong solutions using the energy relation in (3) we calculate the derivative

$$
\frac{d V}{d t}=-\left(\left(\mathcal{D}_{0}+\alpha \mathcal{K}\right) U_{t}, U_{t}\right)+\eta\left[\left\|U_{t}\right\|^{2}-\left(\mathcal{D}_{0} U_{t}, U\right)-(\mathcal{A} U, U)-\varkappa(\mathcal{K} U, U)-(\mathcal{B}(U), U)\right]
$$

Since $\mathcal{D}_{0}$ is bounded from $\bar{H}^{1 / 2}$ into $\bar{H}$, we obtain that

$$
\left|\left(\mathcal{D}_{0} U_{t}, U\right)\right| \leq \varepsilon\left\|\mathcal{A}^{1 / 2} U\right\|^{2}+C \varepsilon^{-1}\left\|U_{t}\right\|^{2}, \quad \forall \varepsilon>0 .
$$

Thus by Assumption 3.4(ii) there exist $b_{i}>0$ independent of $(\alpha, \varkappa)$ such that

$$
\frac{d V}{d t} \leq-\left[\left(\left(\mathcal{D}_{0}+\alpha \mathcal{K}\right) U_{t}, U_{t}\right)-b_{1} \eta\left\|U_{t}\right\|^{2}\right]-b_{2} \eta\left[E_{*}(t)+\varkappa(\mathcal{K} U, U)\right]+\eta b_{3},
$$

This implies that there exists $0<\eta_{*} \leq \eta_{0}$ independent of $(\alpha, \varkappa) \in \Lambda$ such that

$$
\frac{d V_{\beta}}{d t}+b_{2} \eta\left[E_{*}(t)+\varkappa(\mathcal{K} U, U)\right] \leq \eta b_{3}
$$

\footnotetext{
${ }^{2}$ See the Appendix for the notions related with this theorem.
} 
for all $(\alpha, \varkappa) \in \Lambda$ and $\eta \in\left(0, \eta_{*}\right]$, where $V_{\beta}=V+\beta_{1}>0$.

Now we split the parametric region $\Lambda$ into several subdomains.

We start with the following case. Let $\varkappa_{*}>0$ and $\alpha_{*}>\bar{\alpha}$ be fixed. We take $0<\tilde{\varkappa} \leq \varkappa_{*}$ such that $\alpha_{*}>\tilde{\varkappa} / \eta_{*}$ Then we take $\eta=\tilde{\varkappa} \alpha^{-1}$. In this case $\eta \leq \eta_{*}$ and

$$
\mu=\frac{1}{2}(\varkappa+\eta \alpha)=\frac{1}{2}(\varkappa+\tilde{\varkappa}) \leq \varkappa .
$$

Thus for all $\varkappa \geq \varkappa_{*}$ and $\alpha \geq \alpha_{*}$ we have that

$$
\frac{d V_{\beta}}{d t}+b_{2} \eta\left[E_{*}(t)+\mu(\mathcal{K} U, U)\right] \leq \eta b_{3} .
$$

In particular, (9) yields

$$
\frac{d V_{\beta}}{d t}+b_{2} \eta \beta_{2}^{-1} V_{\beta} \leq \eta b_{4} \text { with } b_{4}=b_{3}+\frac{\beta_{1}+\beta_{3}}{\beta_{2}},
$$

where $b_{i}$ and $\beta_{i}$ do not depend on $\alpha$ and $\varkappa$. This implies that

$$
V_{\beta}(t) \leq V_{\beta}(0) e^{-b_{2} \eta \beta_{2}^{-1} t}+\frac{b_{4} \beta_{2}}{b_{2}} .
$$

Since the value $b_{4} b_{2}^{-1} \beta_{2}$ is independent of $\varkappa \geq 0$ and $\alpha \geq \bar{\alpha}$, we can conclude from the previous argument that the set $\mathfrak{B}$ is absorbing for all $\varkappa>0$ and $\alpha>\bar{\alpha}$ with $R$ independent of $\varkappa$ and $\alpha$.

In the case when $\varkappa=0$ from (10) we obtain

$$
\frac{d V_{\beta}}{d t}+b_{2} \eta E_{*}(t) \leq \eta b_{3} .
$$

Now we take $\alpha \geq \eta_{*}^{-1}$ and $\eta=\alpha^{-1}$. In this case $\eta \leq \eta_{*}$ and $\mu=1 / 2$. Therefore the conclusion follows from the estimate $\left\|\mathcal{K}^{1 / 2} U\right\| \leq c\left\|\mathcal{A}^{1 / 2} U\right\|$ by the same argument as above.

In the case $\varkappa=0$ and $\bar{\alpha} \leq \alpha \leq \eta_{*}^{-1}$ the conclusion is obvious.

So the remaining case is $\varkappa>0$ and $\alpha=\bar{\alpha}$. Now we can take $\eta=\min \left\{\eta_{*}, \varkappa \bar{\alpha}^{-1}\right\}$ when $\bar{\alpha}>0$. It is clear that $\mu \leq \varkappa$ for this case. Thus we can argue as above. In the case $\bar{\alpha}=0$ the relation $\mu \leq \varkappa / 2$ holds automatically. This completes the proof Theorem 3.5 .

This theorem and Proposition 3.2 immediately imply the following result on the existence of a global attractor.

Theorem 3.6 Let Assumptions 2.1, 3.1(ii) and 3.4 be in force. Then for every $(\alpha ; \varkappa) \in \Lambda$ the system $\left(\mathcal{H}, S_{t}\right)$ generated by problem (6) possesses a compact global attractor $\mathfrak{A}$. For every full trajectory $Y=\left\{\left(U(t) ; U_{t}(t)\right): t \in \mathbb{R}\right\}$ from the attractor

$$
\left.\sup _{t \in \mathbb{R}}\left\{\left\|U_{t}(t)\right\|^{2}+\left\|\mathcal{A}^{1 / 2} U(t)\right\|^{2}+\varkappa\left\|\mathcal{K}^{1 / 2} U(t)\right\|^{2}\right\}+\int_{-\infty}^{\infty}\left(\mathcal{D}_{0}+\alpha \mathcal{K}\right) U_{t}(\tau), U_{t}(\tau)\right) d \tau \leq R^{2}
$$

for some $R$ independent of $(\alpha ; \varkappa) \in \Lambda$.

Proof. We first apply the standard result on the existence of a global attractor, see Theorem A.1 in the Appendix. This attractor belongs to the set $\mathfrak{B}$ defined in (8). This implies an uniform bound for the supremum in (11). Using the energy relation in (4) we obtain the corresponding bound for the dissipation integral in (11). 


\subsection{Quasi-stability}

The uniform bounds for the attractor given by Theorem 3.6 are not sufficient to perform the large coupling limit $\alpha \rightarrow+\infty$ and/or $\varkappa \rightarrow+\infty$ in the phase state of the system. One of achievements of this section is stronger uniform estimates for the attractor size. For this we apply the quasistability method in the form suggested in [19] (see also [13, 15, 16, 18, 20] and the survey in the recent monograph [12]). This method makes it also possible to proof finite-dimensionality of the attractor and obtain its smoothness properties.

To apply the quasi-stability method we need additional hypotheses concerning the nonlinear forces $B_{i}(u)$.

Assumption 3.7 Assume that

- $B_{i}(u)=\Pi_{i}^{\prime}(u)$ with the functional $\Pi_{i}: H^{1 / 2} \mapsto \mathbb{R}$ which is a Fréchet $C^{3}$-mapping.

- The second $\Pi_{i}^{(2)}(u)$ and the third $\Pi_{i}^{(3)}(u)$ Fréchet derivatives of $\Pi_{i}(u)$ satisfy the conditions

$$
\left|\left\langle\Pi_{i}^{(2)}(u) ; v, v\right\rangle\right| \leq C_{\rho}\left\|A^{\sigma} v\right\|^{2}, \quad v \in H^{1 / 2},
$$

for some $\sigma<1 / 2$, and

$$
\left|\left\langle\Pi_{i}^{(3)}(u) ; v_{1}, v_{2}, v_{3}\right\rangle\right| \leq C_{\rho}\left\|A^{1 / 2} v_{1}\right\|\left\|A^{1 / 2} v_{2}\right\|\left\|v_{3}\right\|, \quad v_{i} \in H^{1 / 2},
$$

for all $u \in H^{1 / 2}$ such that $\left\|A^{1 / 2} u\right\| \leq \rho$, where $\rho>0$ is arbitrary and $C_{\rho}$ is a positive constant. Here above $\left\langle\Pi_{i}^{(k)}(u) ; v_{1}, \ldots, v_{k}\right\rangle$ denotes the value of the derivative $\Pi_{i}^{(k)}(u)$ on elements $v_{1}, \ldots, v_{k}$.

This assumption concerning nonlinear feedback forces $B_{i}(u)$ appeared earlier in the case of systems with nonlinear damping (see [19, p. 98] and also [14]) to cover the case of critical nonlinearities. We note that Assumption 3.7 holds in both cases of the von Karman and Berger models (see [19] p. 156 and p. 160 respectively). Moreover, as it is shown in [19, p.137], this Assumption [3.7 is also true in the case of the coupled $3 \mathrm{D}$ wave equation in a bounded domain $\Omega \subset \mathbb{R}^{3}$ of the form

$$
\begin{array}{ll}
u_{t t}+\sigma_{1} u_{t}-\Delta u+k_{1}(u-v)+\varphi_{1}(u)=f_{1}(x), & \left.u\right|_{\partial \Omega}=0, \\
v_{t t}+\sigma_{2} v_{t}-\Delta v+k_{2}(u-v)+\varphi_{2}(u)=f_{2}(x), & \left.v\right|_{\partial \Omega}=0,
\end{array}
$$

provided $\varphi_{i} \in C^{2}(\mathbb{R})$ possesses the property $\left|\varphi_{i}^{\prime \prime}(s)\right| \leq C(1+|s|)$ for all $s \in \mathbb{R}$, the parameters $\sigma_{i}$ and $k_{i}$ are nonnegative. Thus our abstract model covers the case of $3 \mathrm{D}$ wave dynamics with a critical force term. We refer to Section 4.2 for a further discussion concerning nonlinear wave equations.

Recall that the Fréchet derivatives $\Pi^{(k)}(u)$ of the functional $\Pi$ are symmetric $k$-linear continuous forms on $H^{1 / 2}$ (see, e.g., [6]). Moreover, if $\Pi \in C^{3}$, then $(B(u), v) \equiv\left\langle\Pi^{\prime}(u) ; v\right\rangle$ is $C^{2}$-functional for every fixed $v \in H^{1 / 2}$ and the following Taylor's expansion holds

$$
(B(u+w)-F(u), v)=\left\langle\Pi^{(2)}(u) ; w, v\right\rangle+\int_{0}^{1}(1-\lambda)\left\langle\Pi^{(3)}(u+\lambda w) ; w, w, v\right\rangle d \lambda
$$


for any $u, v \in H^{1 / 2}[6]$. If Assume that $u(t)$ and $z(t)$ belong to the class $C^{1}\left(a, b ; \mathcal{D}\left(\mathcal{A}^{1 / 2}\right)\right)$ for some interval $[a, b] \subseteq \mathbb{R}$. Then, by the differentiation rule for composition of mappings [6] and using the symmetry of the form $\Pi^{(2)}(u)$, we have that

$$
\frac{d}{d t}\left\langle\Pi^{(2)}(u) ; z, z\right\rangle=\left\langle\Pi^{(3)}(u) ; u_{t}, z, z\right\rangle+2\left\langle\Pi^{(2)}(u) ; z, z_{t}\right\rangle .
$$

Therefore from (15) we obtain the following representation which is important in our further considerations:

$$
\left(B_{i}(u(t)+z(t))-B_{i}(u(t)), z_{t}(t)\right)=\frac{d}{d t} Q_{i}(t)+R_{i}(t), \quad t \in[a, b] \subseteq \mathbb{R},
$$

with

$$
Q_{i}(t)=-\frac{1}{2}\left\langle\Pi_{i}^{(2)}(u(t)) ; z(t), z(t)\right\rangle
$$

and

$$
R_{i}(t)=-\frac{1}{2}\left\langle\Pi_{i}^{(3)}(u) ; u_{t}, z, z\right\rangle+\int_{0}^{1}(1-\lambda)\left\langle\Pi_{i}^{(3)}(u+\lambda z) ; z, z, z_{t}\right\rangle d \lambda .
$$

As we will see below the representation in (16) and the hypotheses listed in Assumption [3.7 can be avoided if the nonlinear forces $B_{i}(u)$ are subcritical, i.e.,

$$
\exists \sigma_{0}<1 / 2: \quad\left\|B_{i}\left(u_{1}\right)-B_{i}\left(u_{2}\right) \mid \leq L(\varrho)\right\| \mathcal{A}^{\sigma_{0}}\left(u_{1}-u_{2}\right)\|, \quad \forall\| \mathcal{A}^{1 / 2} u_{i} \| \leq \varrho .
$$

The representation in (16) leads to the following assertion which, in fact, is proved in [19] (see (4.38), p. 99), but without control of the parameters $\alpha$ and $\varkappa$.

Proposition 3.8 Let Assumptions [2.1 and 3.4 be in force. In addition we suppose that either Assumption 3.7 or relation (19) holds. Let $\mathcal{M} \subset \mathcal{H}$ be a bounded forward invariant set with respect to $S_{t}$ and $U^{i}=S_{t} U_{0}^{i}, i=1,2$ be two solutions to ([6) with (different) initial data $U_{0}^{i} \in \mathcal{M}$. Let $Z=U^{1}-U^{2}$. Then there exist $C, \gamma>0$ such that

$$
E_{Z}(t) \leq C E_{Z}(0) e^{-\gamma t}+C \max _{[0, t]}\left\|\mathcal{A}^{\sigma} Z(\tau)\right\|^{2}, \quad \forall t>0,
$$

where $0 \leq \sigma<1 / 2$ and

$$
E_{Z}(t)=\frac{1}{2}\left(\left\|Z_{t}(t)\right\|^{2}+\left\|\mathcal{A}^{1 / 2} Z(t)\right\|^{2}+\varkappa\left\|\mathcal{K}^{1 / 2} Z\right\|^{2}\right) .
$$

If $\mathcal{M}$ is a uniformly bounded in $\mathcal{H}$ with respect to $(\alpha ; \varkappa) \in \Lambda$ and

$$
(\alpha ; \varkappa) \in \Lambda_{\beta} \equiv\{(\alpha ; \varkappa) \in \Lambda: \alpha \leq \beta(1+\varkappa)\}
$$

for some $\beta>0$, then the constants $C, \gamma$ independent of $(\alpha ; \varkappa)$ but can depend on $\beta$.

Proof. We use the same line of argument as in [19] and start with the following relation (which follows from Lemma 3.23 in [19]):

$$
\begin{aligned}
T E_{Z}(T)+\int_{0}^{T} E_{Z}(t) d t \leq & c\left\{\int_{0}^{T}\left(\left(D_{0}+\alpha \mathcal{K}\right) Z_{t}, Z_{t}\right) d t\right. \\
& \left.+\int_{0}^{T}\left|\left(\left(D_{0}+\alpha \mathcal{K}\right) Z_{t}, Z\right)\right| d t+\Psi_{T}\left(U^{1}, U^{2}\right)\right\}
\end{aligned}
$$


for every $T \geq T_{0} \geq 1$, where $c>0$ does not depend on $\alpha, \varkappa, T$ and

$$
\Psi_{T}\left(U^{1}, U^{2}\right)=\left|\int_{0}^{T}\left(G(\tau), Z_{t}(\tau)\right) d \tau\right|+\left|\int_{0}^{T}(G(t), Z(t)) d t\right|+\left|\int_{0}^{T} d t \int_{t}^{T}\left(G(\tau), Z_{t}(\tau)\right) d \tau\right|
$$

with

$$
G(t)=\mathcal{B}\left(U^{1}(t)\right)-\mathcal{B}\left(U^{2}(t)\right) .
$$

Since every point $(\alpha ; \varkappa) \in \Lambda$ belongs to $\Lambda_{\beta}$ for some $\beta>0$. it is sufficient to consider the case when $(\alpha ; \varkappa) \in \Lambda_{\beta}$ for some $\beta$. In this case we have that

$$
\begin{aligned}
\left|\left(\left(D_{0}+\alpha \mathcal{K}\right) Z_{t}, Z\right)\right| & \leq\left(\left(D_{0}+\alpha \mathcal{K}\right) Z_{t}, Z_{t}\right)^{1 / 2}\left(\left(D_{0}+\alpha \mathcal{K}\right) Z, Z\right)^{1 / 2} \\
& \leq C_{\varepsilon, \beta}\left(\left(D_{0}+\alpha \mathcal{K}\right) Z_{t}, Z_{t}\right)+\varepsilon E_{Z}(t)
\end{aligned}
$$

for every $\varepsilon>0$. Thus choosing $\varepsilon$ in an appropriate way we obtain that

$$
T E_{Z}(T)+\int_{0}^{T} E_{Z}(t) d t \leq c_{\beta} \int_{0}^{T}\left(\left(D_{0}+\alpha \mathcal{K}\right) Z_{t}, Z_{t}\right) d t+c_{0} \Psi_{T}\left(U^{1}, U^{2}\right) .
$$

Under Assumption 3.7 we have from Proposition 4.13 in [19] that for any $\varepsilon>0$ and $T>0$ there exist $a(\varepsilon, T)=a_{\mathcal{M}}(\varepsilon, T)$ and $b(\varepsilon, T)=b_{\mathcal{M}}(\varepsilon, T)$ such that

$$
\begin{aligned}
\sup _{t \in[0, T]}\left|\int_{t}^{T}\left(G(\tau), Z_{t}(\tau)\right) d \tau\right| \leq & \int_{0}^{T}\left[\left\|Z_{t}(\tau)\right\|^{2}+\left\|\mathcal{A}^{1 / 2} Z(\tau)\right\|^{2}\right] d \tau \\
& +a(\varepsilon, T) \int_{0}^{T} d(\tau)\left\|\mathcal{A}^{1 / 2} Z(\tau)\right\|^{2} d \tau+b(\varepsilon, T) \sup _{\tau \in[0, T]}\left\|\mathcal{A}^{\sigma} Z(\tau)\right\|^{2}
\end{aligned}
$$

for all $\varepsilon>0$, where $\sigma<1 / 2$ and

$$
d(t) \equiv d\left(t ; U_{1}, U_{2}\right)=\left\|U_{t}^{1}(t)\right\|^{2}+\left\|U_{t}^{2}(t)\right\|^{2} .
$$

Obviously the same relation (21) (even with $a(\varepsilon, T) \equiv 0)$ remains true in the subcritical case (19)). Thus

$$
\Psi_{T}\left(U^{1}, U^{2}\right) \leq \varepsilon \int_{0}^{T} E_{Z}(\tau) d \tau+a(\varepsilon, T) \int_{0}^{T} d(\tau)\left\|\mathcal{A}^{1 / 2} Z(\tau)\right\|^{2} d \tau+b(\varepsilon, T) \sup _{\tau \in[0, T]}\left\|\mathcal{A}^{\sigma} Z(\tau)\right\|^{2}
$$

for every $\varepsilon>0$. From the energy relation we also obtain that

$$
\int_{0}^{T}\left(\left(D_{0}+\alpha \mathcal{K}\right) Z_{t}, Z_{t}\right) d t \leq E_{Z}(0)-E_{Z}(T)+\Psi_{T}\left(U^{1}, U^{2}\right)
$$

Thus after appropriate choice of $\varepsilon$ and $T$ we arrive at the relation

$$
E_{Z}(T) \leq q E_{Z}(0)+a(T) \int_{0}^{T} d(\tau)\left\|\mathcal{A}^{1 / 2} Z(\tau)\right\|^{2} d \tau+b(T) \sup _{\tau \in[0, T]}\left\|\mathcal{A}^{\sigma} Z(\tau)\right\|^{2},
$$

where $q<1$ and all constants depend on $\beta$. This inequality allows us to apply the same procedure as in [19, p.100] to obtain (20). 
Now we are in position to obtain a result on the finiteness of fractal dimension of the attractors and also additional bounds for trajectories from these attractors.

Theorem 3.9 Let Assumptions 2.1 and 3.4 be in force. In addition we suppose that either Assumption 3.7 or relation (19) holds. Then for any $(\alpha ; \varkappa) \in \Lambda$ the following assertions hold:

1. The global attractor $\mathfrak{A}^{\alpha, \varkappa}$ of the system $\left(\mathcal{H}, S_{t}\right)$ generated by (6) has a finite fractal dimension $\operatorname{dim}_{f} \mathfrak{A}$.

2. This attractor $\mathfrak{A}^{\alpha, \varkappa}$ lies in $\bar{H}^{1} \times \bar{H}^{1 / 2}$ and for every full trajectory $Y=\left\{\left(U(t) ; U_{t}(t)\right): t \in \mathbb{R}\right\}$ from the attractor in addition to the bound in (11) we have that

$$
\sup _{t \in \mathbb{R}}\left\{\left\|U_{t t}(t)\right\|^{2}+\left\|\mathcal{A}^{1 / 2} U_{t}(t)\right\|^{2}+\varkappa\left\|\mathcal{K}^{1 / 2} U_{t}(t)\right\|^{2}\right\} \leq R_{1}(\beta)
$$

for some $R_{1}(\beta)$ independent of $(\alpha ; \varkappa) \in \Lambda_{\beta}$, where $\beta>0$ can be arbitrary.

3. The attractors $\mathfrak{A}^{\alpha, \varkappa}$ are upper semicontinuous at every point $\left(\alpha_{*} ; \varkappa_{*}\right) \in \Lambda$, i.e.,

$$
\lim _{n \rightarrow \infty}\left[\sup \left\{\operatorname{dist}_{\mathcal{H}}\left(y, \mathfrak{A}^{\alpha_{*}, \varkappa_{*}}\right): y \in \mathfrak{A}^{\alpha^{n}, \varkappa^{n}}\right\}\right]=0
$$

for every sequence $\left\{\left(\alpha^{n} ; \varkappa^{n}\right)\right\} \subset \Lambda$ such that $\left(\alpha^{n} ; \varkappa^{n}\right) \rightarrow\left(\alpha_{*} ; \varkappa_{*}\right) \in \Lambda$ as $n \rightarrow \infty$.

Proof. By Proposition 3.8 the system $\left(\mathcal{H}, S_{t}\right)$ is quasi-stable on every bounded forward invariant set. Thus we can apply Theorems 3.4.18 and 3.4.19 from [12] to prove the statements 1 and 2 (see also Theorems A.4 and A.5 in the Appendix).

To prove upper semicontinuity property we can use the methods developed in [25, 26]. In particular, we can apply a result due to [26] (see Theorem A.6 in the Appendix). Indeed, let $\left(\alpha^{n} ; \varkappa^{n}\right) \rightarrow\left(\alpha_{*} ; \varkappa_{*}\right) \in \Lambda$ as $n \rightarrow \infty$. In this case it follows from (22) that

$$
\|\mathcal{A} U(t)\|^{2}+\left\|\mathcal{A}^{1 / 2} U_{t}(t)\right\|^{2} \leq C^{2}, \quad \forall t \in \mathbb{R}
$$

for every full trajectory $\left(U(t) ; U_{t}(t)\right)$ from the attractor $\mathfrak{A}^{\alpha_{n}, \varkappa_{n}}$, where $C$ does not depend on $n$. This means that the attractor $\mathfrak{A}^{\alpha_{n}, \varkappa_{n}}$ belongs to the set

$$
\left\{\left(U_{0}, U_{1}\right):\left\|\mathcal{A} U_{0}\right\|^{2}+\left\|\mathcal{A}^{1 / 2} U_{1}\right\|^{2} \leq C^{2}\right\}
$$

which is compact in $\mathcal{H}$. Thus we only need to show the property (ii) in Theorem A.6.

Let $\left(U_{0}^{n} ; U_{1}^{n}\right) \in \mathfrak{A}^{\alpha_{n}, \varkappa_{n}}$ and $\left(U_{0} ; U_{1}\right) \in \mathfrak{A}^{\alpha_{*}, \varkappa_{*}}$. One can see that

$$
\left(Z(t) ; Z_{t}(t)\right)=S_{t}^{\alpha_{n}, \varkappa_{n}}\left(U_{0}^{n} ; U_{1}^{n}\right)-S_{t}^{\alpha_{*}, \varkappa_{*}}\left(U_{0} ; U_{1}\right) \equiv\left(U^{n}(t)-U(t) ; U_{t}^{n}(t)-U_{t}(t)\right)
$$

satisfies the equation

$$
Z_{t t}+\mathcal{A} Z+\left(\mathcal{D}_{0}+\alpha_{*} \mathcal{K}\right) Z_{t}+\varkappa_{*} \mathcal{K} Z=F
$$

where

$$
F=-\left(\alpha_{n}-\alpha_{*}\right) \mathcal{K} U_{t}^{n}-\left(\varkappa_{n}-\varkappa_{*}\right) \mathcal{K} U^{n}-\mathcal{B}\left(U^{n}\right)+\mathcal{B}(U) .
$$

On the attractors we obviously have that

$$
\|F\| \leq c_{1}\left(\left|\alpha_{n}-\alpha_{*}\right|+\left|\varkappa_{n}-\varkappa_{*}\right|\right)+c_{2}\left\|\mathcal{A}^{1 / 2} Z\right\| .
$$


Therefore the standard energy type calculations gives the estimate

$$
\left\|S_{t}^{\alpha_{n}, \varkappa_{n}} Y_{0}^{n}-S_{t}^{\alpha_{*}, \varkappa_{*}} Y_{0}\right\|_{\mathcal{H}} \leq C\left(\left|\alpha_{n}-\alpha_{*}\right|+\left|\varkappa_{n}-\varkappa_{*}\right|+\left\|Y_{0}^{n}-Y_{0}\right\|_{\mathcal{H}}\right) e^{a t}, \quad t>0,
$$

where $Y_{0}^{n}=\left(U_{0}^{n} ; U_{1}^{n}\right)$ and $Y_{0}=\left(U_{0} ; U_{1}\right)$. Thus we can apply Theorem A.6.

Remark 3.10 The results stated in Theorems 2.3 and 3.9 deal with a general model of the form (2) or (6) and thus they can be also applied in the case of several interacting second order in time equations of the form

$$
u_{t t}^{i}+\nu_{i} A u^{i}+D_{0 i} u_{t}^{i}+\alpha \sum_{j=1}^{N} K_{i j} u_{t}^{j}+\varkappa \sum_{j=1}^{N} K_{i j} u^{j}+B_{i}\left(u^{i}\right)=0, \quad i=1, \ldots, N,
$$

under obvious changes in the set of hypotheses concerning the operators in (24).

\subsection{Asymptotic synchronization}

Now we apply the results above to synchronization. We switch on the interaction operators $\mathcal{K}$ of the standard (see, e.g., [8, 24, 31, 36] and the references therein) symmetric form. Moreover we suppose that the damping operator $\mathcal{D}_{0}$ has a diagonal structure. Namely we assume that

$$
\mathcal{K}=\left(\begin{array}{cc}
1 & -1 \\
-1 & 1
\end{array}\right) K, \quad \mathcal{D}_{0}=\left(\begin{array}{cc}
D_{1} & 0 \\
0 & D_{2}
\end{array}\right)
$$

where $K$ is a strictly positive operator in $H$ with domain $\mathcal{D}(K) \supseteq H^{1 / 2}$, the operators $D_{i}$ : $H^{1 / 2} \mapsto H$ are nonnegative. Thus we consider the following problem

$$
\begin{aligned}
& u_{t t}+\nu_{1} A u+D_{1} u_{t}+\alpha K\left(u_{t}-v_{t}\right)+\varkappa K(u-v)+B_{1}(u)=0, \\
& v_{t t}+\nu_{2} A v+D_{2} v_{t}+\alpha K\left(v_{t}-u_{t}\right)+\varkappa K(v-u)+B_{2}(v)=0
\end{aligned}
$$

with initial data

$$
u(0)=u_{0}, \quad u_{t}(0)=u_{1}, \quad v(0)=v_{0}, \quad v_{t}(0)=v_{1} .
$$

All theorems stated above can be applied to this situation.

Our goal is to study asymptotic synchronization phenomena and we are interested in qualitative behavior of the system in the large coupling limit $\varkappa \rightarrow \infty$ (and/or $\alpha \rightarrow \infty$ ). It is clear from the bound of the attractor given in (11) that it is reasonable to assume that $u=v$ in the this limit. Therefore we need to consider a limiting problem of the form

$$
w_{t t}+\nu A w+D w_{t}+B(w)=0, \quad w(0)=w_{0}, \quad w_{t}(0)=w_{1} .
$$

where

$$
\nu=\frac{\nu_{1}+\nu_{2}}{2}, \quad D=\frac{1}{2}\left(D_{1}+D_{2}\right), \quad B(w)=\frac{1}{2}\left(B_{1}(w)+B_{2}(w)\right) .
$$

Obviously the argument above can be applied to system (27) provided the damping operator $D$ is not degenerate. In fact we can easily prove the following assertion. 
Proposition 3.11 Let Assumptions 2.1 (i,iv), and 3.4 (ii) be in force. Let D: $H^{1 / 2} \mapsto H$ be a strictly positive operator. In addition assume that either Assumption 3.7 or relation (19) is valid. Then problem (27) generates a dynamical system in the space $H^{1 / 2} \times H$ possessing a compact global attractor of finite fractal dimension. This attractor is a bounded set in $H^{1} \times H^{1 / 2}$.

Below we show that the attractors $\mathfrak{A}^{\alpha, \varkappa}$ for problems (26) in some sense converge to the attractor of the limiting system (27) when $\varkappa \rightarrow+\infty$.

Theorem 3.12 Let Assumptions [2.1, 3.1 (ii) and 3.4 be in force with $\mathcal{K}$ and $\mathcal{D}_{0}$ of the form given in (25). Then for every $(\alpha ; \varkappa) \in \Lambda$ the system $\left(\mathcal{H}, S_{t}\right)$ generated by problem (26) possesses a compact global attractor $\mathfrak{A}^{\alpha, \varkappa}$. For every full trajectory $Y=\left\{\left(U(t) ; U_{t}(t)\right): t \in \mathbb{R}\right\}$ with $U(t)=(u(t) ; v(t))$ from the attractor

$$
\sup _{t \in \mathbb{R}}\left\{\left\|U_{t}(t)\right\|^{2}+\left\|\mathcal{A}^{1 / 2} U(t)\right\|^{2}+\varkappa\left\|K^{1 / 2}(u(t)-v(t))\right\|^{2}\right\}+\alpha \int_{-\infty}^{+\infty}\left\|U_{t}(\tau)\right\|^{2} d \tau \leq R^{2}
$$

for some $R$ independent of $(\alpha ; \varkappa) \in \Lambda$.

In addition assume that either Assumption 3.7 or relation (19) holds. Then for any $(\alpha ; \varkappa) \in \Lambda$ the following assertions hold:

1. The global attractor $\mathfrak{A}^{\alpha, \varkappa}$ of the system $\left(\mathcal{H}, S_{t}\right)$ generated by (26) has a finite fractal dimension $\operatorname{dim}_{f} \mathfrak{A}$.

2. This attractor $\mathfrak{A}^{\alpha, \varkappa}$ lies in $\bar{H}^{1} \times \bar{H}^{1 / 2}$ and for every full trajectory $Y=\left\{\left(U(t) ; U_{t}(t)\right): t \in \mathbb{R}\right\}$ from the attractor in addition to the bound in (28) we also have that

$$
\sup _{t \in \mathbb{R}}\left\{\left\|U_{t t}(t)\right\|^{2}+\left\|\mathcal{A}^{1 / 2} U_{t}(t)\right\|^{2}+\varkappa\left\|K^{1 / 2}\left(u_{t}(t)-v_{t}(t)\right)\right\|^{2}\right\} \leq R_{1}^{2}(\beta)
$$

for some $R_{1}(\beta)$ independent of $(\alpha ; \varkappa) \in \Lambda_{\beta}$, where $\beta>0$ can be arbitrary.

3. The attractors $\mathfrak{A}^{\alpha, \varkappa}$ are upper semicontinuous at every point $\left(\alpha_{*} ; \varkappa_{*}\right) \in \Lambda$, i.e., (23) is valid for every sequence $\left\{\left(\alpha^{n} ; \varkappa^{n}\right)\right\} \subset \Lambda$ such that $\left(\alpha^{n} ; \varkappa^{n}\right) \rightarrow\left(\alpha_{*} ; \varkappa_{*}\right) \in \Lambda$ as $n \rightarrow \infty$.

4. Let $u \mapsto B_{i}(u)$ be weakly continuous from $H^{1 / 2}$ into some space $H^{-l}, l \geq 0$. Then in the limit $\varkappa \rightarrow \infty$ we have that

$$
\lim _{\varkappa \rightarrow \infty}\left[\sup \left\{\operatorname{dist}_{\mathcal{H}_{\varepsilon}}(Y, \widetilde{\mathfrak{A}}): Y \in \mathfrak{A}^{\alpha, \varkappa}\right\}\right]=0,
$$

where $\mathcal{H}_{\varepsilon}=\bar{H}^{1 / 2-\varepsilon} \times \bar{H}^{1 / 2-\varepsilon}$ and $\widetilde{\mathfrak{A}}=\left\{\left(u_{0} ; u_{0} ; u_{1} ; u_{1}\right):\left(u_{0} ; u_{1}\right) \in \mathfrak{A}\right\}$. Here $\mathfrak{A}$ is the global attractor for the dynamical system generated by (27). Moreover, if instead of weak continuity of $B_{i}$ we assume that $\nu_{1}=\nu_{2}$ and $K$ commutes 3 with $A$, then the convergence in (30) holds in the space $\bar{H}^{1 / 2} \times \bar{H}^{1 / 2-\varepsilon} \subset \mathcal{H}$.

\footnotetext{
${ }^{3}$ We can take $K=A^{\sigma}$ with some $0 \leq \sigma \leq 1 / 2$, for instance.
} 
Proof. All results except the last one easily follows from Theorem 3.9, Thus we need to establish property (30) only. As in [25, 26] we apply contradiction argument.

Assume that (30) is not true. Then there exist sequences $\left\{\varkappa_{n} \rightarrow \infty\right\}$ and $Y_{0}^{n} \in \mathfrak{A}^{\alpha, \varkappa_{n}}$ such that

$$
\operatorname{dist}_{\mathcal{H}_{\varepsilon}}\left(Y_{0}^{n}, \widetilde{\mathfrak{A}}\right) \geq \delta>0, \quad n=1,2, \ldots
$$

Since $Y_{0}^{n} \in \mathfrak{A}^{\alpha, \varkappa_{n}}$, there exists a full trajectory $Y^{n}=\left\{\left(U^{n}(t) ; U_{t}^{n}(t)\right): t \in \mathbb{R}\right\}$ from the attractor $\mathfrak{A}^{\alpha, \varkappa_{n}}$ such that $Y^{n}(0)=Y_{0}^{n}$. It follows from (28) and (29) and also from Aubin-Dubinsky-Lions theorem (see [37], Corollary 4) that the sequence $Y^{n}$ is compact in $C\left(a, b ; \mathcal{H}_{\varepsilon}\right)$ for every $a<b$ and $*$-weakly compact in $L_{\infty}\left(\mathbb{R} ; \bar{H}^{1 / 2} \times \bar{H}^{1 / 2}\right)$. Thus there exists

$$
\widetilde{U}(t)=(u(t) ; v(t)) \in C^{1}\left(\mathbb{R} ; \mathcal{H}_{\varepsilon}\right) \cap L_{\infty}\left(\mathbb{R} ; \bar{H}^{1 / 2} \times \bar{H}^{1 / 2}\right) \text { with } \widetilde{U}_{t} \in L_{\infty}\left(\mathbb{R} ; \bar{H}^{1 / 2} \times \bar{H}^{1 / 2}\right)
$$

such that along a subsequence

$$
\forall a<b: \sup _{t \in[a, b]}\left\{\left\|U_{t}^{n}(t)-\widetilde{U}_{t}(t)\right\|_{1 / 2-\varepsilon}^{2}+\left\|U^{n}(t)-\widetilde{U}(t)\right\|_{1 / 2-\varepsilon}^{2}\right\} \rightarrow 0, \quad n \rightarrow \infty,
$$

and

$$
\left(U^{n}(t) ; U_{t}^{n}(t)\right) \rightarrow\left(\widetilde{U}(t) ; \widetilde{U}_{t}(t)\right) \quad * \text {-weakly in } L_{\infty}\left(\mathbb{R} ; \bar{H}^{1 / 2} \times \bar{H}^{1 / 2}\right), \quad n \rightarrow \infty .
$$

Since $K$ is strictly positive, it follows from (28) and (29) that

$$
\sup _{t \in \mathbb{R}}\left\{\left\|u_{t}^{n}(t)-v_{t}^{n}(t)\right\|^{2}+\left\|u^{n}(t)-v^{n}(t)\right\|^{2}\right\} \leq \frac{R^{2}}{\varkappa_{n}} \rightarrow 0, \quad n \rightarrow \infty .
$$

By interpolation

$$
\begin{aligned}
\sup _{t \in \mathbb{R}}\left\|A^{1 / 2-\varepsilon}\left(u_{t}^{n}(t)-v_{t}^{n}(t)\right)\right\| & \leq C \sup _{t \in \mathbb{R}}\left\{\left(\left\|A^{1 / 2} u_{t}^{n}(t)\right\|+\left\|A^{1 / 2} v_{t}^{n}(t)\right\|\right)^{1-2 \varepsilon}\left\|u_{t}^{n}(t)-v_{t}^{n}(t)\right\|^{2 \varepsilon}\right\} \\
& \leq C \sup _{t \in \mathbb{R}}\left\|u_{t}^{n}(t)-v_{t}^{n}(t)\right\|^{2 \varepsilon} \rightarrow 0, \quad n \rightarrow \infty .
\end{aligned}
$$

Similarly

$$
\sup _{t \in \mathbb{R}}\left\|A^{1 / 2-\varepsilon}\left(u^{n}(t)-v^{n}(t)\right)\right\| \rightarrow 0, \quad n \rightarrow \infty .
$$

Since $u \mapsto B_{i}(u)$ is weakly continuous for $i=1,2$, these observations allow us to make a limit transition in the variational form of equations (26) and conclude that $\widetilde{U}(t)=(u(t) ; u(t))$, where $u(t)$ is a solution to (27). Moreover, $\left(u(t) ; u_{t}(t)\right)$ is a trajectory bounded in $H^{1 / 2} \times H^{1 / 2}$. Thus it belongs to the attractor $\mathfrak{A}$. It is also clear from (31) that

$$
Y_{0}^{n}=\left(U^{n}(0) ; U_{t}^{n}(0)\right) \rightarrow\left(\widetilde{U}(0) ; \widetilde{U}_{t}(0)\right) \in \widetilde{\mathfrak{A}} \text { in } \mathcal{H}_{\varepsilon}
$$

which is impossible.

In the case when $\nu_{1}=\nu_{2}$ and $K$ commutes with $A$ taking sum of equations (26a) and (26b) we can find that

$$
\sup _{t \in \mathbb{R}}\|A(u(t)+v(t))\| \leq C\left(R_{\beta}\right)
$$


for every trajectory $\left(u(t) ; v(t) ; u_{t}(t) ; v_{t}(t)\right.$ from the attractor $\mathfrak{A}^{\alpha, \varkappa}$ with $(\alpha ; \varkappa) \in \Lambda_{\beta}$. Taking the difference of (26a) and (26b) we obtain that

$$
\sup _{t \in \mathbb{R}}\|A z(t)+2 \varkappa K z(t)\| \leq C\left(R_{\beta}\right) \text { with } z(t)=u(t)-v(t)
$$

for fixed $\alpha$. Since $A$ and $K$ commutes,

$$
\begin{aligned}
\|A z+2 \varkappa K z\|^{2} & =\|A z\|^{2}+4 \varkappa^{2}\|K z\|^{2}+4 \varkappa(A z, K z) \\
& =\|A z\|^{2}+4 \varkappa^{2}\|K z\|^{2}+4 \varkappa\left\|A^{1 / 2} K^{1 / 2} z\right\|^{2} \geq\|A z\|^{2} .
\end{aligned}
$$

Thus (32) and (33) yield additional estimate on the attractor:

$$
\sup _{t \in \mathbb{R}}\{\|A u(t)\|+\|A v(t)\|\} \leq C
$$

with the constant $C$ independent of $\varkappa$. This provides us with compactness of $U^{n}(t)$ in the space $C\left([a, b] ; \bar{H}^{1-\varepsilon}\right)$ for every $\varepsilon>0$ and makes it possible to improve the statement in (30).

Remark 3.13 - The result in (30) means that the attractor $\mathfrak{A}^{\alpha, \varkappa}$ becomes "diagonal" in the limit of large intensity parameter $\varkappa$ with fixed or even absent interaction in velocities. Thus the components of the system becomes synchronized in this limit at the level of global attractors. In particular, this implies that every solution $U(t)=(u(t) ; v(t))$ to (26) demonstrates the following synchronization phenomenon:

$$
\left.\forall \varepsilon>0 \exists \varkappa_{*}: \quad \limsup _{t \rightarrow+\infty}\left[\left\|u_{t}(t)-v_{t}(t)\right\|^{2}+\| A^{1 / 2} u(t)-v(t)\right) \|^{2}\right] \leq \varepsilon, \quad \forall \varkappa \geq \varkappa_{*} .
$$

- The same conclusion as in (30) can be obtain in the limit $(\alpha ; \varkappa) \rightarrow+\infty$ inside of $\Lambda_{\beta}$ for some $\beta$. However as one can see from (30) large $\alpha$ is not necessary for asymptotic synchronization. This observation improves the result established in [24] for finite-dimensional systems which requires for synchronization both parameters $\alpha$ and $\varkappa$ to be large.

- The possibility to obtain synchronization for fixed small $\varkappa$ and large $\alpha$ is problematic. The point is that in the case $\varkappa=0$ under appropriate requirement on nonlinear forces $B_{i}$ there are possible two different stationary solutions which demonstrate absence of asymptotic synchronization.

Now we consider the case of identical interacting subsystems, i.e., we assume that

$$
\nu_{1}=\nu_{2} \equiv \nu, \quad D_{1}=D_{2} \equiv D, \quad B_{1}(w)=B_{2}(w) \equiv B(w)
$$

In this case we observe asymptotic synchronization for finite values of $\varkappa$.

Theorem 3.14 Let the hypotheses of Theorem 3.12 and also relations (34) be in force. Assume that $\alpha \in\left[\bar{\alpha}, \alpha_{*}\right]$ for some fixed $\alpha_{*}$. Let

$$
s_{\varkappa}=\inf \left\{\nu(A w, w)+\varkappa(K w, w): w \in H^{1 / 2},\|w\|=1\right\}
$$


There exists $s_{*}=s_{*}\left(\bar{\alpha}, \alpha_{*}\right)$ and $\omega>0$ such that under the condition $s_{\varkappa} \geq s_{*}$ the property of asymptotic exponential synchronization holds, i.e.,

$$
\left.\lim _{t \rightarrow \infty}\left\{e^{\omega t}\left[\left\|u_{t}(t)-v_{t}(t)\right\|^{2}+\| A^{1 / 2} u(t)-v(t)\right) \|^{2}\right]\right\}=0
$$

for every solution $U(t)=(u(t) ; v(t))$ to (26). In this case $\mathfrak{A}^{\alpha, \varkappa} \equiv \widetilde{\mathfrak{A}}$ for all $\varkappa$ such that $s_{\varkappa} \geq s_{*}$.

Proof. In the case considered $w=u-v$ satisfies the equation

$$
w_{t t}+\nu A w+D w_{t}+2 \alpha K w_{t}+2 \varkappa K w+B(u)-B(v)=0, \quad w(0)=w_{0}, \quad w_{t}(0)=w_{1} .
$$

where $w_{0}=u_{0}-v_{0}$ and $w_{1}=u_{1}-v_{1}$.

We consider the case of the critical nonlinearity, the subcritical case is much simpler. In the former case we use the representation (16) with $z=w$. Since $B_{1}=B_{2}=B$, below we omit the subscript $i$. It follows from (12) and (13) that the variables $Q$ and $R$ defined in (17) and (18) admits the estimates

$$
|Q(t)| \leq C_{R}\left\|A^{\sigma} w(t)\right\|^{2} \text { and }|R(t)| \leq C_{R}\left(\left\|u_{t}(t)\right\|+\left\|v_{t}(t)\right\|\right)\left\|A^{1 / 2} w(t)\right\|^{2}
$$

under the condition

$$
\left\|A^{1 / 2} u(t)\right\|^{2}+\left\|A^{1 / 2} v(t)\right\|^{2} \leq R^{2}
$$

with $R$ and thus $C_{R}$ independent of $\alpha$ and $\varkappa$.

We consider a Lyapunov type function of the form

$$
\Psi(t)=\widetilde{E}(t)+\Phi(t)
$$

where

$$
\widetilde{E}(t)=\frac{1}{2}\left(\left\|w_{t}(t)\right\|^{2}+\nu\left\|A^{1 / 2} w(t)\right\|^{2}\right)+Q(t)
$$

and

$$
\Phi(t)=\eta\left(w, w_{t}\right)+\mu(K w, w)
$$

where $\eta$ is a positive constant which will be chosen later and $\mu=\varkappa+\eta \alpha$. By uniform dissipativity of the system $\left(\mathcal{H}, S_{t}^{\alpha, \varkappa}\right)$ we can assume that that (136) holds with the same $R$ as in (8) for all $t \geq t_{*}$.

One can see that there exists $0<\eta_{0}<1$ and $\beta_{i}>0$ independent of $(\alpha ; \varkappa)$ such that

$$
\beta_{0}\left[E_{0}(t)+\varkappa\left\|K^{1 / 2} w(t)\right\|^{2}-c_{R}\|w(t)\|^{2}\right] \leq \Psi \leq \beta_{2}\left[E_{0}(t)+c_{R}\|w(t)\|^{2}\right]+\mu\left\|K^{1 / 2} w(t)\right\|^{2},
$$

for all $t \geq t_{*}$ and $0<\eta<\eta_{0}$, where

$$
E_{0}(t)=\frac{1}{2}\left(\left\|w_{t}(t)\right\|^{2}+\nu\left\|A^{1 / 2} w(t)\right\|^{2}\right) .
$$

Now on strong solutions we calculate the derivative

$$
\begin{aligned}
\frac{d \Psi}{d t}= & -\left((D+\alpha K) w_{t}, w_{t}\right)-R(t) \\
& +\eta\left[\left\|w_{t}\right\|^{2}-\left(D w_{t}, w\right)-\nu(A w, w)-2 \varkappa(K w, w)-(B(u)-B(v), w)\right]
\end{aligned}
$$

\footnotetext{
${ }^{4}$ One can see that $s_{\varkappa} \geq \varkappa \cdot \inf \operatorname{spec}(K)$. Thus if $K$ is not degenerate, then $s_{\varkappa} \rightarrow+\infty$ as $\varkappa \rightarrow+\infty$.
} 
Since $D$ is bounded from $H^{1 / 2}$ into $H$, we obtain that

$$
\left|\left(D w_{t}, w\right)\right| \leq \varepsilon\left\|A^{1 / 2} w\right\|^{2}+C \varepsilon^{-1}\left\|w_{t}\right\|^{2}, \quad \forall \varepsilon>0 .
$$

Thus there exist $b_{i}>0$ independent of $(\alpha, \varkappa)$ such that

$$
\begin{aligned}
\frac{d \Psi}{d t} \leq & -\left[\left((D+\alpha K) w_{t}, w_{t}\right)-b_{1} \eta\left\|w_{t}\right\|^{2}\right]+C_{R}\left(\left\|u_{t}\right\|+\left\|v_{t}\right\|\right)\left\|A^{1 / 2} w\right\|^{2} \\
& -b_{2} \eta\left[E_{0}(t)+\varkappa\left\|K^{1 / 2} w\right\|^{2}\right]+\eta c_{R}\|w(t)\|^{2}
\end{aligned}
$$

Fixing $\alpha$ and taking $s_{\varkappa}$ large enough we obtain that

$$
\frac{d \Psi}{d t}+\omega \Psi(t)-C_{R}\left(\left\|u_{t}\right\|^{2}+\left\|v_{t}\right\|^{2}\right)\left\|A^{1 / 2} w\right\|^{2} \leq 0, \quad t \geq t_{*},
$$

for some $\omega, C>0$. Using finiteness of the dissipativity integrals:

$$
\int_{0}^{\infty}\left(\left\|u_{t}\right\|^{2}+\left\|v_{t}\right\|^{2}\right) d t<\infty
$$

we obtain (35). The equality $\mathfrak{A}^{\alpha, \varkappa} \equiv \widetilde{\mathfrak{A}}$ for the attractors follows from (35).

If $B$ is critical, but not satisfies the structural hypothesis in Assumption 3.7 we can still guarantee asymptotic exponential synchronization. However in this case we need additional condition that the damping parameter $\alpha$ is large enough and $K$ is not degenerate. If $B$ are globally Lipschitz we can even avoid the requirement of dissipativity of the system.

Remark 3.15 The results similar to Theorems 3.12 and 3.14 can be also established for $N$ coupled second order in time equations of the form

$$
\begin{aligned}
& u_{t t}^{1}+\nu_{1} A u^{1}+D_{1} u_{t}^{1}+\alpha K\left(u_{t}^{1}-u_{t}^{2}\right)+\varkappa K\left(u^{1}-u^{2}\right)+B_{1}\left(u^{1}\right)=0, \\
& u_{t t}^{j}+\nu_{j} A u^{j}+D_{i} u_{t}^{j}-\alpha K\left(u_{t}^{j+1}-2 u_{t}^{j}+u_{t}^{j-1}\right)-\varkappa K\left(u^{j+1}-2 u^{j}+u^{j-1}\right)+B_{j}\left(u^{j}\right)=0 \text {, } \\
& j=2, \ldots, N-1, \\
& u_{t t}^{N}+\nu_{N} A u^{N}+D_{N} u_{t}^{N}+\alpha K\left(u_{t}^{N}-u_{t}^{N-1}\right)+\varkappa K\left(u^{N}-u^{N-1}\right)+B_{N}\left(u^{N}\right)=0,
\end{aligned}
$$

This system can be reduced to (6) with

$$
\mathcal{D}_{0}=\left(\begin{array}{ccccc}
D_{1} & 0 & 0 & \ldots & 0 \\
0 & D_{2} & 0 & \ldots & 0 \\
0 & 0 & D_{3} & \ldots & 0 \\
\vdots & \vdots & \vdots & \ddots & \vdots \\
0 & 0 & 0 & \ldots & D_{N}
\end{array}\right), \quad \mathcal{K}=\left(\begin{array}{ccccc}
1 & -1 & 0 & \ldots & 0 \\
-1 & 2 & -1 & \ldots & 0 \\
0 & -1 & 2 & \ldots & 0 \\
\vdots & \vdots & \vdots & \ddots & \vdots \\
0 & 0 & 0 & \ldots & 1
\end{array}\right) K .
$$

Thus the general results of this section can be applied with the same hypotheses concerning the operators $A, D_{i}, K$ and $B_{i}$. We note that the energy for for this $N$ coupled model has the form

$$
\mathcal{E}=\sum_{j=1}^{N}\left[\frac{1}{2}\left(\left\|u_{t}^{j}\right\|^{2}+\nu_{j}\left\|A^{1 / 2} u^{j}\right\|^{2}\right)+\Pi_{j}\left(u^{j}\right)\right]+\frac{\varkappa}{2} \sum_{j=1}^{N-1}\left\|K^{1 / 2}\left(u^{j+1}-u^{j}\right)\right\|^{2} .
$$


In the ODE case $\left(\nu_{i} \equiv 0, K=i d\right)$ synchronization for this model was considered in [24] with assumption that both $\alpha$ and $\varkappa$ become large or even tend to infinity. Our approach allows us observe asymptotic synchronization for fixed $\alpha$ and in the limit $\varkappa \rightarrow+\infty$ (for identical subsystems it is sufficient to assume that $\varkappa$ is large enough). The limiting (synchronized) regime is described by problem (27) with

$$
\nu=\frac{1}{N} \sum_{j=1}^{N} \nu_{j}, \quad D=\frac{1}{N} \sum_{j=1}^{N} D_{j}, \quad B(w)=\frac{1}{N} \sum_{j=1}^{N}\left(B_{j}(w) .\right.
$$

In the case of a plate with the Berger nonlinearity the same result was obtained in [32] with $D_{0 j}=d_{j} \cdot i d, \alpha=0$ and $K=i d$.

\subsection{On synchronization by means of finite-dimensional coupling}

One can see from the argument given in Theorem 3.14 that asymptotic synchronization can be achieved even with finite-dimensional coupling operator. Indeed, the only condition we need is

$$
\nu(A w, w)+\varkappa(K w, w) \geq c\|w\|^{2}, \quad \forall w \in H^{1 / 2},
$$

with appropriate $c$ depending on the size of an absorbing ball. As it was already mentioned if $K$ is a strictly positive operator, then the requirement $s_{\varkappa} \geq s_{*}$ holds for large intensity parameter $\varkappa$. However it is not necessary to assume non-degeneracy of the operator $K$ to guarantee large $s_{\varkappa}$. For instance, if $K=P_{N}$ is the orthoprojector onto $\operatorname{Span}\left\{e_{k}: k=1,2, \ldots, N\right\}$, then

$$
\begin{aligned}
& \nu(A w, w)+\varkappa(K w, w) \geq \sum_{k=1}^{N}\left(\nu \lambda_{k}+\varkappa\right)\left|\left(w, e_{k}\right)\right|^{2}+\nu \sum_{k=N+1}^{\infty} \lambda_{k}\left|\left(w, e_{k}\right)\right|^{2} \\
& \geq\left(\nu \lambda_{1}+\varkappa\right) \sum_{k=1}^{N}\left|\left(w, e_{k}\right)\right|^{2}+\nu \lambda_{N+1} \sum_{k=N+1}^{\infty}\left|\left(w, e_{k}\right)\right|^{2} \geq \min \left\{\nu \lambda_{1}+\varkappa, \nu \lambda_{N+1}\right\}\|w\|^{2} .
\end{aligned}
$$

Thus if $\varkappa \geq \nu\left(\lambda_{N+1}-\lambda_{1}\right)$, then we can guarantee large $s_{\varkappa}$ by an appropriate choice of $N$.

This fact admits some generalization which based on an assumption that $K$ is a "good" approximation (in some sense) for a strictly positive operator.

Let $V \subset H$ be separable Hilbert spaces and $K, L$ be linear operators from $V$ into $H$. Assume that $L$ is a strictly positive on $V$, i.e., there exists $a_{L}>0$ such that

$$
(L u, u) \geq a_{L}\|u\|_{H}^{2}, \quad u \in V .
$$

We introduce the value

$$
e(L, K) \equiv e_{V}^{H}(L, K)=\sup \left\{\|L u-K u\|_{H}:\|u\|_{V} \leq 1\right\} .
$$

In the case when $L=i d$ is the identity operator this value is known (see [2]) as the global approximation error in $H$ arising in the approximation of elements $v \in V$ by elements $K v$.

Now we take $V=H^{1 / 2}$. It follows from the definition that

$$
\|L u-K u\|_{H} \leq e(L, K)\left\|A^{1 / 2} u\right\|, \quad u \in H^{1 / 2} .
$$


In this case we obtain

$$
\begin{aligned}
\nu(A w, w)+\varkappa(K w, w) & =\nu\left\|A^{1 / 2} w\right\|^{2}+\varkappa(L w, w)+\varkappa((K-L) w, w) \\
& \geq \nu\left\|A^{1 / 2} w\right\|^{2}+a_{L} \varkappa\|w\|^{2}-\varkappa e(L, K)\|w\|\left\|A^{1 / 2} w\right\| \\
& \geq\left(a_{L} \varkappa-\frac{\varkappa^{2} e^{2}(L, K)}{4 \nu}\right)\|w\|^{2}
\end{aligned}
$$

Thus according Theorem 3.14 under the condition

$$
a_{L} \varkappa-\frac{\varkappa^{2} e^{2}(L, K)}{4 \nu} \geq s_{*}
$$

we have asymptotic exponential synchronization. The latter inequality is valid, when $e^{2}(L, K) \leq$ $2 \nu a_{L} \varkappa^{-1}$ and $\varkappa \geq 2 s_{*} a_{L}^{-1}$ for instance. So $e(L, K)$ should be small and $\varkappa$ large. We note that in the case $L=i d$ and $K=P_{N}$ we have $e(L, K)=\lambda_{N+1}^{-1 / 2}$ and $a_{L}=1$. So the inequalities above can be realized for some choice $\varkappa$ and $N$.

Now we describe another situation where synchronization can be achieved with finite-dimensional coupling. For this we use interpolation operators related with a finite family $\mathcal{L}$ of linear continuous functionals $\left\{l_{j}: j=1, \ldots, N\right\}$ on $H^{1 / 2}$. Following [9] (see also [19, 20]) we introduce the notion of completeness defect of a set $\mathcal{L}$ of linear functionals on $H^{1 / 2}$ with respect to $H$ by the formula

$$
\epsilon_{\mathcal{L}} \equiv \epsilon_{\mathcal{L}}\left(H^{1 / 2}, H\right)=\sup \left\{\|w\|_{H}: w \in H^{1 / 2}, l(w)=0, l \in \mathcal{L},\|w\|_{1 / 2} \leq 1\right\} .
$$

It is clear that $\epsilon_{\mathcal{L}_{1}} \geq \epsilon_{\mathcal{L}_{2}}$ provided $\operatorname{Span} \mathcal{L}_{1} \subset \operatorname{Span} \mathcal{L}_{2}$ and $\epsilon_{\mathcal{L}}=0$ if and only if the class of functionals $\mathcal{L}$ is complete in $H^{1 / 2}$, i.e, the property $l(w)=0$ for all $l \in \mathcal{L}$ implies $w=0$. For further properties of the completeness defect we refer to [9, Chapter 5].

We define the class $\mathcal{R}_{\mathcal{L}}$ of so-called interpolation operators which are related with the set of functionals given. We say that a operator $K$ belongs to $\mathcal{R}_{\mathcal{L}}$ if it has the form

$$
K v=\sum_{j=1}^{N} l_{j}(v) \psi_{j}, \quad \forall v \in H^{1 / 2},
$$

where $\left\{\psi_{j}\right\}$ is an arbitrary finite set of elements from $H^{1 / 2}$. An operator $K \in \mathcal{R}_{\mathcal{L}}$ is called Lagrange interpolation operator, if it has form (38) with $\left\{\psi_{j}\right\}$ such that $l_{k}\left(\psi_{j}\right)=\delta_{k j}$. In the case of Lagrange operators we have that $l_{j}(u-K u)=0$ and thus (37) yields

$$
\|u-K u\| \leq \epsilon_{\mathcal{L}}\|v\|_{1 / 2}, \quad v \in H^{1 / 2} .
$$

Hence the smallness of the completeness defect is important requirement from point of view of synchronization. We refer to [9, Chapter 5] for properties of this characteristic and for description of sets of functionals with small $\epsilon_{\mathcal{L}}$. The simplest example is modes. In this case $\mathcal{L}=\left\{l_{j}(u)=\right.$ $\left.\left(u, e_{j}\right): j=1,2, \ldots, N\right\}$, where $\left\{e_{k}\right\}$ are eigenfunctions of the operator $A$. The operator $P_{\mathcal{L}}$ given by

$$
P_{\mathcal{L}} v=\sum_{j=1}^{N}\left(e_{j}, v\right) e_{j}, \quad \forall v \in H^{1 / 2},
$$


is the Lagrange interpolation operator. Moreover, $\epsilon_{\mathcal{L}}=e\left(i d, P_{\mathcal{L}}\right)=\lambda_{N+1}^{-1 / 2}$. Thus the completeness defect (and the global approximation error) can be made small after an appropriate choice of $N$. This shows that the situation with $K=P_{N}$ can be included in the general framework.

Unfortunately in the general case an interpolation operator of the form (38) is not symmetric and positive. Therefore formally we cannot apply the result on uniform dissipativity with $K$ of the form (38). The situation requires a separate consideration and possibly another set of hypotheses concerning the model. We plan to provide with the corresponding analysis in future. Here we give only one particular result in this direction.

We consider the following version of the equations (26)

$$
\begin{gathered}
u_{t t}+\nu A u+D u_{t}+\varkappa K(u-v)+B(u)=0, \\
v_{t t}+\nu A v+D v_{t}+\varkappa K(v-u)+B(v)=0, \\
u(0)=u_{0}, \quad u_{t}(0)=u_{1}, \quad v(0)=v_{0}, \quad v_{t}(0)=v_{1} .
\end{gathered}
$$

Theorem 3.16 Assume that A satisfies Assumption 2.1(i), B(u) is globally Lipschitz and subcritical, i.e.,

$$
\exists \sigma_{0}<1 / 2: \quad\left\|B\left(u_{1}\right)-B\left(u_{2}\right) \mid \leq L_{B}\right\| \mathcal{A}^{\sigma_{0}}\left(u_{1}-u_{2}\right) \|, \quad \forall u_{i} \in H^{1 / 2} .
$$

Let $D: H^{1 / 2} \mapsto H$ be strictly positive operator and $K \equiv K_{\mathcal{L}}$ be a Lagrange interpolation operator for some family $\mathcal{L}$ of linear continuous functionals $\left\{l_{j}: j=1, \ldots, N\right\}$ on $H^{1 / 2}$. Then for every initial data $U_{0}=\left(u_{0} ; v_{0}\right) \in \bar{H}^{1 / 2}$ and $U_{1}=\left(u_{1} ; v_{1}\right) \in \bar{H}$ problem (39) has unique generalized solution $U(t)=(u(t) ; v(t))$ and there exist $\varkappa_{*}>0$ and $\epsilon_{*}(\varkappa)$ such that for every $\varkappa \geq \varkappa_{*}$ and $\epsilon_{\mathcal{L}} \leq \epsilon_{*}(\varkappa)$ the solution $U(t)=(u(t) ; v(t))$ is asymptotically synchronized, i.e., relation (35) holds with some $\omega>0$.

Proof. This is globally Lipschitz case and therefore the well-posedness easily follows from [19, Theorem 1.5].

Following the same idea as in Theorem 3.14 we find that the difference $w=u-v$ satisfies the equation

$$
w_{t t}+\nu A w+D w_{t}+2 \varkappa w+G_{\varkappa, \mathcal{L}}(u, v)=0, \quad w(0)=w_{0}, \quad w_{t}(0)=w_{1} .
$$

where $w_{0}=u_{0}-v_{0}, w_{1}=u_{1}-v_{1}$ and

$$
G_{\varkappa, \mathcal{L}}(u, v)=-2 \varkappa\left(i d-K_{\mathcal{L}}\right)(u-v)+B(u)-B(v) .
$$

We have the obvious estimate

$$
\left\|G_{\varkappa, \mathcal{L}}(u, v)\right\| \leq 2 \varkappa \epsilon_{\mathcal{L}}\left\|A^{1 / 2} w\right\|+L_{B}\left\|\mathcal{A}^{\sigma_{0}} w\right\| \leq 2\left(\varkappa \epsilon_{\mathcal{L}}+\delta\right)\left\|A^{1 / 2} w\right\|+C_{\delta}\|w\|
$$

for all $\delta>0$. This implies

$$
\left|\left(G_{\varkappa, \mathcal{L}}(u, v), w\right)\right| \leq\left(\varkappa \epsilon_{\mathcal{L}}+\delta\right)^{2}\left\|A^{1 / 2} w\right\|^{2}+C_{\delta}\|w\|^{2}, \quad \forall \delta>0,
$$

and

$$
\left|\left(G_{\varkappa, \mathcal{L}}(u, v), w_{t}\right)\right| \leq \mu\left\|w_{t}\right\|^{2}+\frac{2\left(\varkappa \epsilon_{\mathcal{L}}+\delta\right)^{2}}{\mu}\left\|A^{1 / 2} w\right\|^{2}+C_{\delta, \mu}\|w\|^{2}, \quad \forall \mu, \delta>0 .
$$


Now as in the proof of Theorem 3.14 we can use Lyapunov type functional

$$
\Psi\left(w, w_{t}\right)=\frac{1}{2}\left(\left\|w_{t}\right\|^{2}+\nu\left\|A^{1 / 2} w\right\|^{2}\right)+\varkappa\left\|w_{t}\right\|^{2}+\eta\left(w, w_{t}\right),
$$

with $\varkappa$ large, $\varkappa \epsilon_{\mathcal{L}}$ small and with an appropriate choice of $\mu$ and $\delta$.

\section{Applications}

In this section we shortly outline possible application of the general results presented above.

\subsection{Plate models}

We first consider the plate models with coupling via elastic (Hooke type) links. Namely, we consider the following PDEs

$$
\begin{aligned}
& u_{t t}+\gamma_{1} u_{t}+\Delta^{2} u+\varkappa K(u-v)+\varphi_{1}(u)=f_{1} \text { in } \Omega \subset \mathbb{R}^{2}, \\
& v_{t t}+\gamma_{2} v_{t}+\Delta^{2} v+\varkappa K(v-u)+\varphi_{2}(u)=f_{2} \text { in } \Omega \subset \mathbb{R}^{2},
\end{aligned}
$$

with the hinged boundary conditions

$$
u=\Delta u=0, \quad v=\Delta v=0 \text { on } \partial \Omega,
$$

where $K$ is an operator which will be specified later. The nonlinear force term $\varphi_{i}(u)$ can take one of the following forms:

- Kirchhoff model: $\varphi \in \operatorname{Lip}_{\text {loc }}(\mathbb{R})$ fulfills the condition

$$
\liminf _{|s| \rightarrow \infty} \frac{\varphi(s)}{s}>-\lambda_{1}^{2},
$$

where $\lambda_{1}$ is the first eigenvalue of the Laplacian with the Dirichlet boundary conditions.

- Von Karman model: $\varphi(u)=\left[u, v(u)+F_{0}\right]$, where $F_{0}$ is a given function in $H^{4}(\Omega)$ and the bracket $[u, v]$ is given by

$$
[u, v]=\partial_{x_{1}}^{2} u \cdot \partial_{x_{2}}^{2} v+\partial_{x_{2}}^{2} u \cdot \partial_{x_{1}}^{2} v-2 \cdot \partial_{x_{1} x_{2}}^{2} u \cdot \partial_{x_{1} x_{2}}^{2} v .
$$

The Airy stress function $v(u)$ solves the following elliptic problem

$$
\Delta^{2} v(u)+[u, u]=0 \text { in } \Omega, \quad \frac{\partial v(u)}{\partial n}=v(u)=0 \text { on } \partial \Omega .
$$

Von Karman equations are well known in nonlinear elasticity and describes nonlinear oscillations of a plate accounting for large displacements, see [20, 29] and the references therein.

- Berger Model: In this case the feedback force has the form

$$
\varphi(u)=-\left[\kappa \int_{\Omega}|\nabla u|^{2} d x-\Gamma\right] \Delta u,
$$

where $\kappa>0$ and $\Gamma \in \mathbb{R}$ are parameters, for some details and references see [9, Chap.4]. 
In all these cases we have that $H=L^{2}(\Omega)$ and

$$
A u=\Delta^{2} u, \quad u \in \mathcal{D}(\mathcal{A})=\left\{u \in H^{4}(\Omega): u=\Delta u=0 \text { on } \partial \Omega\right\} .
$$

It is clear that $A$ satisfies Assumption 2.1 (i) and $\mathcal{D}\left(A^{1 / 2}\right)=H^{2}(\Omega) \cap H_{0}^{1}(\Omega)$.

The nonlinear force $\varphi$ in the Kirchhoff model is subcritical with respect to the energy space (it is locally Lipschitz from $H^{1+\delta}(\Omega)$ into $L_{2}(\Omega)$ for every $\delta>0$ ). In contrast, the von Karman and Berger nonlinearities are critical (they are locally Lipschitz mappings from $H^{2}(\Omega)$ into $L_{2}(\Omega)$ which are not compact on $H^{2}(\Omega)$ ). Other requirements concerning the corresponding forcing terms $B_{i}$ can be verified in the standard way. For details we refer to [14] for the Kirchhoff forces, to [19, Chapter 6] for the von Karman model and to [19, Chapter 7] for the case of Berger plates.

The interaction operator $K$ can be of the following forms $K=i d$ and $K=-\Delta$, or even $K=A^{\sigma}$ with $0<\sigma<1 / 2$. In the purely Kirchhoff case with globally Lipschitz functions $\varphi_{i}$ we can also use the Lagrange interpolation operator with respect to nodes, i.e., with respect to the family of functionals

$$
l_{j}(w)=w\left(x_{j}\right), \text { where } x_{j} \in \Omega, \quad j=1, \ldots, N,
$$

with appropriatt 5 choice of nodes $x_{j}$. This means that two Kirchhoff plates can be synchronized by finite number of point links.

We note that in the case when both $\varphi_{1}$ and $\varphi_{2}$ are Berger nonlinearities (possibly with different parameters) the results on synchronization with $K=i d$ can be found in [31, see also [32, 33.

We also mention some other plate models for which the abstract results established can be applied:

- First of all we can consider the plates with other (self-adjoint) boundary conditions such as clamped and free and also combinations of them (for a discussion of these boundary conditions in the case of nonlinear plate models we refer to [20]).

- The plate models with rotational inertia can be included in the framework presented. Instead of (40) coupled dynamics in these models can be described by equations of the form

$$
\begin{aligned}
& (1-\gamma \Delta) u_{t t}+\mu(1-\gamma \Delta) u_{t}+\Delta^{2} u+\varkappa K(u-v)+\varphi_{1}(u)=f_{1} \text { in } \Omega \subset \mathbb{R}^{2}, \\
& (1-\gamma \Delta) v_{t t}+\mu(1-\gamma \Delta) v_{t}+\Delta^{2} v+\varkappa K(v-u)+\varphi_{2}(u)=f_{2} \text { in } \Omega \subset \mathbb{R}^{2} .
\end{aligned}
$$

Here $\gamma$ is positive. It is convenient to rewrite (41) as equations in $H=H_{0}^{1}(\Omega)$ (equipped with the inner product $\left.(u, v)_{H} \equiv((1-\gamma \Delta) u, v)_{L^{2}(\Omega)}\right)$ in the form (26) with the operator $A$ generated by the form $a(u, v)=(\Delta u, \Delta v)_{L^{2}(\Omega)}$ on $H_{0}^{2}(\Omega)$.

- We can also include into consideration the plates with Kirchhoff-Boussinesq forces of the form

$$
\varphi(u)=-\operatorname{div}\left\{|\nabla u|^{2} \nabla u\right\}+a|u|^{q} u
$$

with $a, q \geq 0$, see [19, Chapter 7] and also [17, 21] concerning models with this force.

\footnotetext{
${ }^{5}$ For details concerning smallness of the corresponding completeness defect we refer to [9, Chapter 5].
} 


\subsection{Coupled wave equations}

In the case of coupled wave equations (14) the standard (critical) hypotheses concerning the source term $\varphi \in C^{2}(\mathbb{R})$ in the $3 \mathrm{D}$ case have the form:

$$
\liminf _{|s| \rightarrow \infty}\left\{\varphi_{i}(s) s^{-1}\right\}>-\lambda_{1}, \quad\left|\varphi_{i}^{\prime \prime}(s)\right| \leq C(1+|s|), s \in \mathbb{R},
$$

where $\lambda_{1}$ is the first eigenvalue of the minus Laplacian with the Dirichlet boundary conditions, see, e.g., [19, Chapter 5] for details.

We can also consider several versions of damped sine-Gordon equations. These are used to model the dynamics of Josephson junctions driven by a source of current (see, e.g., [39] for comments and references). For instance, we can consider the system 6

$$
\begin{aligned}
& u_{t t}+\gamma u_{t}-\Delta u+\beta u+\varkappa(u-v)+\lambda \sin u=f(x), \\
& v_{t t}+\gamma v_{t}-\Delta v+\beta v+\varkappa(v-u)+\lambda \sin v=f(x),
\end{aligned}
$$

in a smooth domain $\Omega \subset \mathbb{R}^{d}$ and equipped with the Neumann boundary conditions

$$
\left.\frac{\partial u}{\partial n}\right|_{\partial \Omega}=0,\left.\quad \frac{\partial v}{\partial n}\right|_{\partial \Omega}=0 .
$$

It is easy to see that in the case of the Dirichlet boundary conditions the general theory developed above can be applied. The same is true when $\beta>0$. In the case $\beta=0$ the situation is more complicated because the corresponding operator $A$ is $-\Delta$ on the domain

$$
\mathcal{D}(A)=\left\{u \in H^{2}(\Omega): \frac{\partial u}{\partial n}=0 \text { on } \partial \Omega\right\}
$$

and thus becomes degenerate. So we concentrate on the case $\beta=0$.

It is convenient to introduce new variables

$$
w=\frac{u-v}{2} \text { and } z=\frac{u+v}{2} .
$$

In these variables problem (42) with $\beta=0$ can be written in the form

$$
\begin{aligned}
& w_{t t}+\gamma w_{t}-\Delta w+2 \varkappa w+\lambda \sin w \cos z=0, \\
& z_{t t}+\gamma z_{t}-\Delta z+\lambda \cos w \sin z=f(x), \\
& \left.\frac{\partial w}{\partial n}\right|_{\partial \Omega}=0,\left.\quad \frac{\partial z}{\partial n}\right|_{\partial \Omega}=0 .
\end{aligned}
$$

The main linear part in (44a) is not degenerate when $\varkappa>0$. Therefore the same calculations as in the proof of Theorem 3.14 shows that there exists $\varkappa_{*}$ such that

$$
\exists \eta>0: \quad\|w(t)\|_{H^{1}(\Omega)}^{2}+\left\|w_{t}(t)\right\|^{2} \leq C_{B} e^{-\eta t}, \quad t>0,
$$

\footnotetext{
${ }^{6}$ For simplicity we discuss a symmetric coupling of identical systems only.
} 
when $\varkappa \geq \varkappa_{*}$ for all initial data from a bounded set $B$ in $H^{1}(\Omega) \times L_{2}(\Omega)$. This means that every trajectory is asymptotically synchronized. Moreover, it follows from the reduction principle (see [12, Section 2.3.3]) that the limiting (synchronized) dynamics is determined by the single equation

$$
z_{t t}+\gamma z_{t}-\Delta z+\lambda \sin z=f(x),\left.\quad \frac{\partial z}{\partial n}\right|_{\partial \Omega}=0
$$

The long-time dynamics of this equation is described in [39, Chapter 4]. We also refer to [27, 28] for the studies of synchronization phenomena for (42) in the homogeneous (ODE) case.

Another coupled sine-Gordon systems of an interest is the following one

$$
\begin{aligned}
& u_{t t}+\gamma u_{t}-\Delta u+\lambda \sin (u-v)=f_{1}(x), \\
& v_{t t}+\gamma v_{t}-\Delta v+\lambda \sin (v-u)=f_{2}(x), \\
& \left.u\right|_{\partial \Omega}=0,\left.\quad v\right|_{\partial \Omega}=0 .
\end{aligned}
$$

Formally this model is out of the scope the theory developed above. However, using the ideas presented we can answer some questions concerning synchronized regimes 7

In variables $w$ and $z$ given by (43) we have equations

$$
\begin{aligned}
& w_{t t}+\gamma w_{t}-\Delta w+\lambda \sin 2 w=g(x), \\
& z_{t t}+\gamma z_{t}-\Delta z=h(x), \\
& \left.w\right|_{\partial \Omega}=0,\left.\quad z\right|_{\partial \Omega}=0,
\end{aligned}
$$

where $g(x)=\left(f_{1}(x)-f_{2}(x)\right) / 2$ and $h(x)=\left(f_{1}(x)+f_{2}(x)\right) / 2$. One can see that

$$
\left\|z_{t}(t)\right\|^{2}+\left\|\nabla\left(z(t)-z_{*}\right)\right\|^{2} \leq C\left(\left\|z_{t}(0)\right\|^{2}+\left\|\nabla\left(z(0)-z_{*}\right)\right\|^{2}\right) e^{-\omega t}, \quad t \geq 0,
$$

for some $C, \omega>0$, where $z_{*} \in H^{1}(\Omega)$ solves the Dirichlet problem

$$
-\Delta z=h(x),\left.\quad z\right|_{\partial \Omega}=0 .
$$

Problem (46a) equipped with the Dirichlet boundary conditions possesses a compact global attractor $\mathfrak{A}$ in the space $W=H_{0}^{1}(\Omega) \times L_{2}(\Omega)$, see [39, Chapter 4]. Hence

$$
\left(\begin{array}{c}
u(t)-z_{*} \\
u_{t}(t) \\
v(t)-z_{*} \\
v_{t}(t)
\end{array}\right)=\left(\begin{array}{c}
z(t)-z_{*} \\
z_{t}(t) \\
z(t)-z_{*} \\
z_{t}(t)
\end{array}\right)+\left(\begin{array}{c}
w(t) \\
w_{t}(t) \\
-w(t) \\
-w_{t}(t)
\end{array}\right) \rightarrow\left\{\left(\begin{array}{c}
\psi \\
-\psi
\end{array}\right): \psi \in \mathfrak{A}\right\} \quad \text { as } t \rightarrow+\infty
$$

in the space $W \times W$. Thus we observe some kind of shifted asymptotic anti-phase synchronization.

\section{A Appendix: Some facts from the theory of dynamical systems}

In this section we collect several definitions and general theorems from the theory of dissipative dynamical systems. For more complete presentation we refer to one of the monographs [3, 9, 23, 39 .

\footnotetext{
${ }^{7}$ In the ODE case the synchronization phenomena in (45]) was studied in [27, 28].
} 
Recall that a dynamical system (see, e.g., [9, 23, 39]) is a pair $\left(X, S_{t}\right)$ of a complete metric space $X$ and a family of continuous mappings $S_{t}: X \mapsto X, t \geq 0$, satisfying the semigroup property: (i) $S_{t+\tau}=S_{t} \circ S_{\tau}$ for any $t, \tau \geq 0$, and (ii) $S_{0}$ is the identity operator. It is also assumed that $t \mapsto S_{t} x$ is continuous mapping for every $x \in X$.

A system $\left(X, S_{t}\right)$ is said to be dissipative if it possesses a bounded absorbing set $B$. A closed set $B \subset X$ is said to be absorbing for $S_{t}$ if for any bounded set $D \subset X$ there exists $t_{0}(D)$ such that $S_{t} D \subset B$ for all $t \geq t_{0}(D)$. If the phase space $X$ is a Banach space, then the radius of a ball containing an absorbing set is called a radius of dissipativity of the system.

A system $\left(X, S_{t}\right)$ is said to be asymptotically smooth if for any closed bounded set $B \subset X$ such that $S_{t} B \subset B$ for all $t \geq 0$ there exists a compact set $\mathcal{K}=\mathcal{K}(B)$ which uniformly attracts $B$, i.e.,

$$
\lim _{t \rightarrow+\infty} \sup \left\{\operatorname{dist}\left(S_{t} y, \mathcal{K}\right): y \in B\right\}=0 .
$$

A system is called gradient if it possesses a strict Lyapunov function. The latter is defined as a continuous functional $\Phi(x)$ on $X$ satisfying (i) $\Phi\left(S_{t} x\right) \leq \Phi(x)$ for all $t \geq 0$ and $x \in X$, and (ii) if $\Phi(x)=\Phi\left(S_{t} x\right)$ for all $t>0$, then $x$ is a stationary point of $S_{t}$, i.e., $S_{t} x=x$ for all $t \geq 0$.

A global attractor of a dynamical system $\left(X, S_{t}\right)$ is a bounded closed set $\mathfrak{A} \subset X$ which is invariant (i.e., $S_{t} \mathfrak{A}=\mathfrak{A}$ ) and uniformly attracts all other bounded sets:

$$
\lim _{t \rightarrow \infty} \sup \left\{\operatorname{dist}\left(S_{t} y, \mathfrak{A}\right): y \in B\right\}=0 \quad \text { for any bounded set } B \text { in } X .
$$

It is known 3, 9, 23, 39 that the global attractor consists of all bounded full trajectories. We recall that a curve $\{y(t): t \in \mathbb{R}\} \subset X$ is said to be a full trajectory if $S_{t} y(\tau)=y(t+\tau)$ for all $\tau \in \mathbb{R}$ and $t \geq 0$.

The standard criterion (see, e.g., [9, 23, 39]) for the existence of a global attractor is the following assertion.

Theorem A.1 Let $\left(X, S_{t}\right)$ be a dissipative asymptotically smooth dynamical system on a complete metric space $X$. Then $S_{t}$ possesses a unique compact global attractor $\mathfrak{A}$ such that

$$
\mathfrak{A}=\omega\left(B_{0}\right)=\bigcap_{t>0} \overline{\bigcup_{\tau \geq t} S_{\tau} B_{0}}
$$

for every bounded absorbing set $B_{0}$.

For gradient systems it is also useful the following criterion of the existence of a global attractor (see, e.g., [35, Theorem 4.6]).

Theorem A.2 Let $\left(X, S_{t}\right)$ be an asymptotically smooth gradient system which has the property that for any bounded set $B \subset X$ there exists $\tau>0$ such that $\gamma_{\tau}(B) \equiv \bigcup_{t \geq \tau} S_{t} B$ is bounded. If the set $\mathcal{N}$ of stationary points is bounded, then $\left(X, S_{t}\right)$ has a compact global attractor $\mathfrak{A}$ which coincides with the unstable set $\mathbb{M}_{+}(\mathcal{N})$ emanating from $\mathcal{N}$, i.e., $\mathfrak{A}=\mathbb{M}_{+}(\mathcal{N})$.

We recall (see, e.g., [3]) that the unstable set $\mathbb{M}_{+}(\mathcal{N})$ emanating from $\mathcal{N}$ is a subset of $X$ such that for each $z \in \mathbb{M}_{+}(\mathcal{N})$ there exists a full trajectory $\{y(t): t \in \mathbb{R}\}$ satisfying $u(0)=z$ and $\operatorname{dist}(y(t), \mathcal{N}) \rightarrow 0$ as $t \rightarrow-\infty$. 
Remark A.3 We note that the hypothesis that $\gamma_{\tau}(B)$ is bounded in Theorem A.2 can be changed in the following (additional) requirements concerning the Lyapunov function $\Phi(x)$ : (i) $\Phi(x)$ is bounded from above on any bounded set, and (ii) the set $\Phi_{R}=\{x \in X: \Phi(x) \leq R\}$ is bounded for every $R$ (see, e.g., [19, Corollary 2.29]).

An important feature of a global attractor is its (fractal) dimension. We recall that the fractal dimension $\operatorname{dim}_{f}^{X} M$ of a compact set $M$ in a complete metric space $X$ is defined (see, e.g., [39]) as

$$
\operatorname{dim}_{f}^{X} M=\limsup _{\varepsilon \rightarrow 0} \frac{\ln N(M, \varepsilon)}{\ln (1 / \varepsilon)},
$$

where $N(M, \varepsilon)$ is the minimal number of closed sets in $X$ of diameter $2 \varepsilon$ needed to cover the set $M$.

Now we state several facts related with asymptotically quasi-stable systems (for details we refer to the recent monograph [12] and the references therein).

Let $X$ and $Y$ be reflexive Banach spaces; $X$ is compactly embedded in $Y$. We endow the space $H=X \times Y$ with the norm

$$
\|y\|_{H}^{2}=\left\|u_{0}\right\|_{X}^{2}+\left\|u_{1}\right\|_{Y}^{2}, \quad y=\left(u_{0} ; u_{1}\right) .
$$

Assume that $\left(H, S_{t}\right)$ is a dynamical system with the evolution operator of the form

$$
S_{t} y=\left(u(t) ; u_{t}(t)\right), \quad y=\left(u_{0} ; u_{1}\right) \in H,
$$

where the function $u(t)$ possesses the property

$$
u \in C\left(\mathbb{R}_{+}, X\right) \cap C^{1}\left(\mathbb{R}_{+}, Y\right) .
$$

A dynamical system $\left(H, S_{t}\right)$ with an evolution operator of the form (47) is said to be asymptotically quasi-stable on a set $\mathcal{B} \subset H$ if there exist a compact seminorm $\mu_{X}(\cdot)$ on the space $X$ and nonnegative scalar functions $a(t), b(t)$, and $c(t)$ on $\mathbb{R}_{+}$such that (i) $a(t)$ and $c(t)$ are locally bounded on $[0, \infty)$, (ii) $b(t) \in L_{1}\left(\mathbb{R}_{+}\right)$possesses the property $\lim _{t \rightarrow \infty} b(t)=0$, and (iii) for every $y_{1}, y_{2} \in \mathcal{B}$ and $t>0$ the following relations

$$
\left\|S_{t} y_{1}-S_{t} y_{2}\right\|_{H}^{2} \leq a(t) \cdot\left\|y_{1}-y_{2}\right\|_{H}^{2}
$$

and

$$
\left\|S_{t} y_{1}-S_{t} y_{2}\right\|_{H}^{2} \leq b(t) \cdot\left\|y_{1}-y_{2}\right\|_{H}^{2}+c(t) \cdot \sup _{0 \leq s \leq t}\left[\mu_{X}\left(u^{1}(s)-u^{2}(s)\right)\right]^{2}
$$

hold. Here we denote $S_{t} y_{i}=\left(u^{i}(t) ; u_{t}^{i}(t)\right), i=1,2$. The theory of quasi-stable systems was started with [15] and has been developed in [12, 19, 20, 22]. The main outcome of this theory is the following assertion.

Theorem A.4 (Global attractor) Assume that the system $\left(H, S_{t}\right)$ is dissipative and asymptotically quasi-stable on a bounded forward invariant absorbing set $\mathcal{B}$ in $H$. Then the system $\left(H, S_{t}\right)$ possesses a compact global attractor $\mathfrak{A}$ of finite fractal dimension.

Another consequence of quasi-stability is the following assertion which states some regularity of the attractor and provides additional bounds for trajectories in it. 
Theorem A.5 (Regularity) Assume that the dynamical system $\left(H, S_{t}\right)$ possesses a compact global attractor $\mathfrak{A}$ and is asymptotically quasi-stable on $\mathfrak{A}$. Moreover, we assume that (48) holds with the function $c(t)$ possessing the property $c_{\infty}=\sup _{t \in \mathbb{R}_{+}} c(t)<\infty$. Then any full trajectory $\left\{\left(u(t) ; u_{t}(t)\right): t \in \mathbb{R}\right\}$ that belongs to the global attractor enjoys the following regularity properties

$$
u_{t} \in L_{\infty}(\mathbb{R} ; X) \cap C(\mathbb{R} ; Y), \quad u_{t t} \in L_{\infty}(\mathbb{R} ; Y) .
$$

Moreover, there exists $R>0$ such that

$$
\left\|u_{t}(t)\right\|_{X}^{2}+\left\|u_{t t}(t)\right\|_{Y}^{2} \leq R^{2}, \quad t \in \mathbb{R}
$$

where $R$ depends on the constant $c_{\infty}$, on the seminorm $\mu_{X}$, and also on the embedding properties of $X$ into $Y$.

In the study of synchronization phenomena we deal with stability of attractors with respect to interaction intensity. Variations of the intensity parameter is treated as a perturbation of the dynamical system. To describe these variations at the abstract level we consider a family of dynamical systems $\left(X, S_{t}^{\lambda}\right)$ with the same phase space $X$ and with evolution operators $S_{t}^{\lambda}$ depending on a parameter $\lambda$ from a complete metric space $\Lambda$. The following assertion is proved by Kapitansky and Kostin [26] (see also [3] and [23] for related results).

Theorem A.6 (Upper semicontinuity) Assume that a dynamical system $\left(X, S_{t}^{\lambda}\right)$ in a complete metric space $X$ possesses a compact global attractor $\mathfrak{A}^{\lambda}$ for every $\lambda \in \Lambda$. Assume that the following conditions hold.

(i) There exists a compact $K \subset X$ such that $\mathfrak{A}^{\lambda} \subset K$.

(ii) If $\lambda_{k} \rightarrow \lambda_{0}, x_{k} \rightarrow x_{0}$ and $x_{k} \in \mathfrak{A}^{\lambda_{k}}$, then

$$
S_{\tau}^{\lambda_{k}} x_{k} \rightarrow S_{\tau}^{\lambda_{0}} x_{0} \quad \text { for some } \tau>0 .
$$

Then the family $\left\{\mathfrak{A}^{\lambda}\right\}$ of attractors is upper semicontinuous at the point $\lambda_{0}$; that is,

$$
d_{X}\left\{\mathfrak{A}^{\lambda} \mid \mathfrak{A}^{\lambda_{0}}\right\} \equiv \sup \left\{\operatorname{dist}_{X}\left(x, \mathfrak{A}^{\lambda_{0}}\right): x \in \mathfrak{A}^{\lambda}\right\} \rightarrow 0 \text { as } \lambda \rightarrow \lambda_{0} .
$$

Moreover, if (49) holds for every $\tau>0$, then the upper limit $\mathfrak{A}\left(\lambda_{0}, \Lambda\right)$ of the attractors $\mathfrak{A}^{\lambda}$ at $\lambda_{0}$ defined by the formula

$$
\mathfrak{A}\left(\lambda_{0}, \Lambda\right)=\bigcap_{\delta>0} \overline{\bigcup\left\{\mathfrak{A}^{\lambda}: \lambda \in \Lambda, 0<\operatorname{dist}\left(\lambda, \lambda_{0}\right)<\delta\right\}}
$$

is a nonempty compact strictly invariant set lying in the attractor $\mathfrak{A}^{\lambda_{0}}$ and possessing the property

$$
d_{X}\left\{\mathfrak{A}^{\lambda} \mid \mathfrak{A}\left(\lambda_{0}, \Lambda\right)\right\} \rightarrow 0 \text { as } \lambda \rightarrow \lambda_{0}
$$




\section{References}

[1] V. Afraimovich, S-N. Chow, J. Hale, Synchronization in lattices of coupled oscillators. Physica $D, 103$ (1997), 44-451.

[2] J.-P. Aubin, Approximation of Elliptic Boundary-Value Problems, Wiley, New York, 1972.

[3] A.V. Babin, M.I. Vishik, Attractors of Evolution Equations, North-Holland, Amsterdam, 1992.

[4] A. Balanov, N.Janson, D. Postnov, O. Sosnovtseva, Synchronization: From Simple to Complex, Berlin-Heidelberg, Springer, 2008.

[5] T. Caraballo, I. Chueshov, P. Kloeden, Synchronization of a stochastic reaction-diffusion system on a thin two-layer domain, SIAM J. Math. Anal. 38, (2007), 1489-1507.

[6] H. Cartan, Calculus Différentielles, Hermann, Paris, 1967.

[7] A. Carvalho, M. R. T. Primo, Boundary synchronization in parabolic problems with nonlinear boundary conditions, Dynamics Cont. Discr. Impul. Systems 7 (2000), 541-560.

[8] A. Carvalho, H. Rodrigues, T. Dlotko, Upper semicontinuity of attractors and synchronization, J. Math. Anal. Appl., 220 (1998), 13-41.

[9] I. Chueshov, Introduction to the Theory of Infinite-Dimensional Dissipative Systems. Acta, Kharkov, 1999 (in Russian); English translation: Acta, Kharkov, 2002; http://www.emis.de/monographs/Chueshov/.

[10] I. Chueshov, A reduction principle for coupled nonlinear parabolic-hyperbolic PDE, J. Evol. Equ., 4 (2004), 591-612.

[11] I. Chueshov, Invariant manifolds and nonlinear master-slave synchronization in coupled systems, Appl. Anal., 86 (2007), 269-286.

[12] I.Chueshov, Dynamics of Quasi-Stable Dissipative Systems, Springer, New York, 2015.

[13] I. Chueshov, M. Eller, I. Lasiecka, Finite dimensionality of the attractor for a semilinear wave equation with nonlinear boundary dissipation. Commun. PDEs, 29 (2004) 1847-1876.

[14] I. Chueshov, S. Kolbasin, Plate models with state-dependent damping coefficient and their quasi-static limits, Nonlin. Anal. TMA, 73 (2010), 1626-1644.

[15] I. Chueshov, I. Lasiecka, Attractors for second-order evolution equations with a nonlinear damping, J. Dyn. Dif. Eqs, 16 (2004), 469-512.

[16] I. Chueshov, I. Lasiecka, Global attractors for von Karman evolutions with a nonlinear boundary dissipation, J. Dif. Eqs, 198 (2004), 196-221.

[17] I. Chueshov, I. Lasiecka, Existence, uniqueness of weak solutions and global attractors for a class of nonlinear 2D Kirchhoff-Boussinesq models, Discr. Cont. Dyn. Sys., 15 (2006), 777-809. 
[18] I. Chueshov, I. Lasiecka, Long-time dynamics of von Karman semi-flows with nonlinear boundary/interior damping, J. Dif. Eqs, 233 (2007), 42-86.

[19] I. Chueshov, I. Lasiecka. Long-Time Behavior of Second Order Evolution Equations with Nonlinear Damping. Memoirs of AMS 912, AMS, Providence, 2008.

[20] I. Chueshov, I. Lasiecka, Von Karman Evolution Equations, Springer, New York, 2010.

[21] I. Chueshov, I. Lasiecka, On global attractor for 2D Kirchhoff-Boussinesq model with supercritical nonlinearity, Commun. PDEs, 36 (2011), 67-99.

[22] I. Chueshov, I. Lasiecka, Well-posedness and long time behavior in nonlinear dissipative hyperbolic-like evolutions with critical exponents. In: Nonlinear Hyperbolic PDEs, Dispersive and Transport Equations, HCDTE Lecture Notes, Part I, G. Alberti et al. (Eds.), AIMS on Applied Mathematics, vol.6, AIMS, Springfield, 2013, 1-96.

[23] J.K. Hale, Asymptotic Behavior of Dissipative Systems, AMS, Providence, RI, 1989.

[24] J. Hale, Diffusive coupling, dissipation, and synchronization, J. Dyn. Dif. Eqs, 9 (1997), $1-52$.

[25] J. Hale, X. Lin, G. Raugel, Upper semicontinuity of attractors for approximations of semigroups and partial differential equations, Math. Comp. 50 (1988), 89-1-23.

[26] L.V. Kapitansky, I.N. Kostin, Attractors of nonlinear evolution equations and their approxiamtins, Leningrad Math. J., 2 (1991), 97-117.

[27] G. Leonov, V. Reitmann, V. Smirnova, Non-Local Methods for Pendulum-Like Feedback Systems, Stuttgart-Leipzig, Teubner, 1992.

[28] G. Leonov, V. Smirnova, Mathematical Problems of Phase Synchronization Theory, St. Petersburg, Nauka, 2000; in Russian.

[29] J. L. Lions, Quelques Méthodes de Résolution des Problèmes aux Limites Non Linéaires, Dunod, Paris, 1969.

[30] E. Mosekilde, Y. Maistrenko, D. Postnov. Chaotic Synchronization, World Scientific Publishing Co., River Edge, NJ, 2002.

[31] O Naboka, Synchronization of nonlinear oscillations of two coupling Berger plates, Nonlin. Anal., TMA, 67 (2007), 1015-1026.

[32] O Naboka, Synchronization phenomena in the system consisting of m coupled Berger plates, J. Math. Anal. Appl., 341 (2008), 1107-1124.

[33] O Naboka, On synchronization of oscillations of two coupled Berger plates with nonlinear interior damping, Commun. Pure Appl. Anal., 8 (2009), 1933-1956.

[34] G. Osipov, J. Kurths, C. Zhou Synchronization in Oscillatory Networks, Berlin-Heidelberg, Springer, 2007. 
[35] G. Raugel, Global attractors in partial differential equations, in: Handbook of Dynamical Systems, vol. 2, Elsevier Sciences, Amsterdam, 2002, pp.885-992.

[36] H. Rodrigues, Abstract methods for synchronization and applications, Appl. Anal., 62 (1996), 263-296.

[37] J. Simon, Compact sets in the space $L^{p}(0, T ; B)$, Annali Mat. Pura Appl., 148 (1987), 65-96.

[38] S. Strogatz, Sync, Hyperion Books, New York, 2003.

[39] R. Temam, Infinite-Dimensional Dynamical Dystems in Mechanics and Physics, Springer, New York, 1988.

[40] C. W. Wu, Synchronization in coupled chaotic circuits and systems, World Scientific Publishing Co., River Edge, NJ, 2002. 